\title{
Nonadiabatic Electron Response in the Hasegawa-Wakatani Equations
}

T. Stoltzfus-Dueck, ${ }^{1, \text { a) }}$ B. D. Scott, ${ }^{2}$ and J. A. Krommes ${ }^{3}$

${ }^{1)}$ Max-Planck-Institut für Plasmaphysik, EURATOM Association, Teilinstitut Greifswald, Wendelsteinstr. 1, 17491 Greifswald, Germany

2) Max-Planck-Institut für Plasmaphysik, EURATOM Association, Boltzmannstraße 2, 85748 Garching, Germany

3) PPPL, Princeton University, P.O. Box 451, MS 28, Princeton, NJ 08543-0451

(Dated: September 30, 2013)

Tokamak edge turbulence is strongly influenced by parallel electron physics, which relaxes density and potential fluctuations towards electron adiabatic response. Beginning with the paradigmatic Hasegawa-Wakatani equations (HWEs) for resistive tokamak edge turbulence, a unique decomposition of the electric potential $(\varphi)$ into adiabatic $(a)$ and nonadiabatic $(b)$ portions is derived, based on the requirement that $a$ neither drive nor respond to the parallel current $j_{\|}$. The form of the decomposition clarifies that, at perpendicular scales large relative to the sound radius, the electron adiabatic response controls the nonzonal $\varphi$, not the fluctuating density $n$. Simple energy balance arguments allow one to rigorously bound the ratio of rms nonzonal nonadiabatic fluctuations $(\tilde{b})$ relative to adiabatic ones $(\tilde{a})$. The role of the vorticity nonlinearity in transferring energy between adiabatic and nonadiabatic fluctuations aids intuitive understanding of self-sustained turbulence in the HWEs. When the normalized parallel resistivity is weak, $\tilde{b}$ becomes effectively slaved, allowing the reduction to an approximate one-field model that remains valid for strong turbulence. In addition to guiding physical intuition, the one-field reduction should greatly ease further analytical manipulations. Direct numerical simulation of the 2D HWEs confirms the convergence of the asymptotic formula for $\tilde{b}$.

\section{INTRODUCTION}

Physics in the confined steep-gradient region just inside the last closed flux surface (LCFS) of toroidal confinement devices plays a critical role in setting the boundary conditions for the core plasma. If core transport is "stiff," as some experimental evidence ${ }^{1,2}$ and theoretical models ${ }^{3-5}$ suggest, then the temperature at the magnetic axis should scale linearly with the pedestal temperature. ${ }^{6}$ Also, the favorable H-mode confinement regime ${ }^{7}$ required for the success of ITER, appears to have its origins in the physics of the edge region, although its detailed mechanism remains an open question to this day. ${ }^{8,9}$ While the complexity of edge physics requires numerical efforts for quantitative modeling, physical understanding is enhanced by the development of simple analytical models, which may capture basic features and scalings of the turbulence and aid understanding of both numerical and experimental results, as well as suggest fruitful new directions for inquiry. Since the nonlinear behavior of edge turbulence may be quite different from the linear behavior, ${ }^{10}$ with some models even exhibiting self-sustained turbulence in the absence of linear instabilities, ${ }^{11-13}$ it is important that reduced modeling not be too closely tied to linear mode structure.

Edge turbulence differs significantly from core turbulence due to the strong radial gradients and resulting extreme anisotropy between parallel and perpendicular length scales, ${ }^{14}$ which make the nonadiabatic passing electron response nonnegligible. ${ }^{15}$ Concurrently, the com-

\footnotetext{
a) Electronic mail: tstoltzf@ipp.mpg.de
}

bination of poloidal and toroidal periodicity with magnetic shear and strong nonlinearity ensures $k_{\|} \gtrsim 1 / q R$ for most of the fluctuations, ${ }^{16,17}$ so that electron parallel response remains important. ${ }^{15}$ The two-field collisional drift turbulence model of Hasegawa and Wakatani ${ }^{18,19}$ represents one of the simplest systems to span this intermediate regime between adiabatic and hydrodynamic electrons, allowing the turbulence to nonlinearly, selfconsistently determine the relation between electron density and electric potential. While statistical treatments of two-field plasma turbulence models have enjoyed reasonable success ${ }^{20-22}$ including successful prediction of particle flux levels in the Hasegawa-Wakatani equations (HWEs), ${ }^{23}$ the resulting statistical moment equations are relatively complex, motivating the reduction to a one-field model. The paradigm Hasegawa-Mima (HM) equation $^{24}$ enforces adiabatic electron response, thus missing not only energy growth and damping but also the $\boldsymbol{E} \times \boldsymbol{B}$ nonlinearity. Many one-field generalizations reincorporate these quantities, almost always by assuming a specified linear relationship between electron density and potential, leading to so-called $i \delta$ models. ${ }^{25-27}$ However, numerical simulations in disagreement with the linear density-potential relationship assumed in such approaches ${ }^{10,28}$ provide good motivation for attempts to extend one-field models to nonlinear density-potential relationships. Previous $i \delta$-like generalizations have retained one of the full time-derivatives contributing to the adiabatic density ${ }^{29}$ and allowed the frequency in the formula to be nonlinearly shifted. ${ }^{30}$ Crotinger and Dupree (CD) nonlinearly extended a linear collisionless densitypotential relation by replacing the linear frequency with nonlinear time derivatives evaluated using the HasegawaMima equation, effectively broadening the frequency as well as shifting it. ${ }^{31}$ Using a similar iterative procedure, 
Naulin and Spatschek (NS) obtained a one-field nearadiabatic reduction of the $2 \mathrm{D}$ HWEs. ${ }^{32}$ While these reductions to one-field models represent significant simplification, the iteration involved in the more faithful models results in lengthy, complicated equations with terms that are difficult to interpret physically.

In the spirit of the more systematic reductions, ${ }^{31,32}$ the present work derives a near-adiabatic one-field reduction of the HWEs, physically resting on rapid parallel electron motion and slow parallel ion motion relative to the frequency scales of the perpendicular drifts. The central new contribution is an exact variable transform decomposing the electrostatic potential into uniquely defined adiabatic and nonadiabatic portions, which makes iteration unnecessary. This leads to a much simpler one-field model, both physically transparent and amenable to further analytic manipulation, that remains valid for fullynonlinear strong turbulence. Additionally, the quadratic invariants in the new variables may be used to set an upper bound on the level of nonadiabaticity for nondecaying turbulence, demonstrating convergence of the approximation. The presented transformation and asymptotic reduction may also be applied to fuller model equations, incorporating electromagnetic fluctuations and X-point geometry, as will be presented in upcoming publications.

This paper is laid out as follows. Section II presents a simple discussion of the collisional drift wave instability, clarifying the physics addressed by this paper and explaining why, at larger scales, it is the potential rather than the density that is slaved by the electron adiabatic response. Section III executes the variable transformation that underlies all of the following analysis. Connections of the new decomposition's variables with those of previous authors are highlighted. Section IV discusses the nonlinear quadratic invariants in the new variables. A bound on the level of nonadiabaticity in nondecaying turbulence is derived. Possible application to selfsustained turbulence is discussed. Section V systematically derives the one-field near-adiabatic approximation to the HWEs that constitutes the principal result of this work. Parameter regimes appropriate for its application are estimated. Section VI presents direct numerical simulation verifying the derived bounds, convergence of the near-adiabatic approximation, and lack of convergence of its $i \delta$-like linearization. Finally, Section VII summarizes the results of the paper.

\section{BASIC DRIFT WAVE PHYSICS}

In tokamak edge turbulence, the characteristic times for parallel electron response are often shorter than those of cross-field drifts, while parallel ion times are always much longer. ${ }^{15}$ When parallel electron response is fast, one expects electrons to approach parallel force balance, usually referred to in plasma physics as electron adiabatic response. However, nonadiabatic passing electrons generally play an important role in edge turbulence, so one

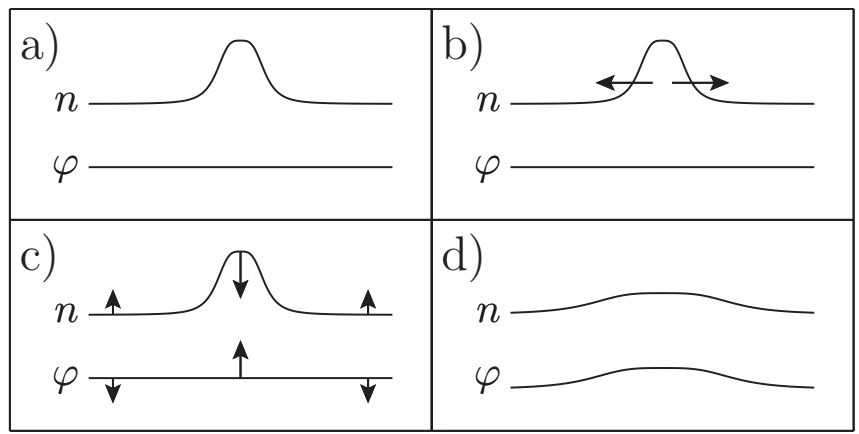

Figure 1. Schematic of parallel electron response: a) Electrons out of parallel force balance. b) Parallel electron flux is excited. c) Density and potential respond to parallel electron flux. d) Parallel electron force balance is restored.

must carefully consider the approach of electrons toward adiabatic response.

If one takes the component of electron force balance along the magnetic field and neglects electron inertia, collisions, and trapping, the electron pressure and electric forces are left to balance each other: $0=-\nabla_{\|} p_{e}-n_{e} e E_{\|}$. If one assumes electrons to be isothermal, neglects electromagnetic effects, and considers small-amplitude fluctuations, this may be rewritten as $T_{e 0} \nabla_{\|} n_{e}=n_{e 0} e \nabla_{\|} \phi$ or, using the dimensionless forms $n \doteq\left(n_{e}-n_{e 0}\right) / n_{e 0}$ and $\varphi \doteq e \phi / T_{e 0}$, as $\nabla_{\|} n=\nabla_{\|} \varphi{ }^{33}$ It is in fact just this balance that motivates the standard normalizations.

What happens if this force balance is disturbed? Consider a case in which electrons are out of parallel force balance, for example the density hump sketched in Fig. 1a. Due to the unbalanced pressure force, electrons will flow out of the hump (Fig. 1b). The outflux of electrons causes a reduced density in the hump region. However, the electron outflux is also a parallel outflux of negative charge. Ions polarization-drift out of the hump, across the magnetic field, to almost perfectly balance the electron outflux, but the electrostatic potential must increase somewhat in order to excite the cross-field ion flux (Fig. 1c). The decrease in electron density and increase in electric potential both act to restore parallel electron force balance (Fig. 1d). The collisional electrostatic limit of this physics, due in general to shear and kinetic Alfvén responses, ${ }^{34}$ is captured by the HWEs treated here. ${ }^{18,19}$

Notably, this simple analysis has not identified which of $n$ or $\varphi$ changed more in order to restore parallel electron force balance. To answer this, one must turn to the quasineutrality equation which, neglecting curvature contributions, dominantly balances parallel electron current against perpendicular ion polarization current: $0=\nabla \cdot \boldsymbol{j} \approx \nabla_{\|} j_{\|}+\nabla_{\perp} \cdot\left(n_{i} e \boldsymbol{u}_{i, \mathrm{pol}}\right)$. Assuming small-amplitude and small-scale fluctuations and a single species of cold, singly ionized ions, this relation may be linearized and simplified to $\partial_{t} \rho_{s}^{2} \nabla_{\perp}^{2} \varphi=\nabla_{\|}\left(j_{\|} / n_{e 0} e\right)$, where $\rho_{s} \doteq c_{s} / \Omega_{c i}$ is the "sound radius," $c_{s} \doteq \sqrt{T_{e 0} / m_{i}}$ is the cold-ion sound speed, and $\Omega_{c i} \doteq e B_{0} / m_{i} c$ is the 
ion gyrofrequency. The density response to the parallel electron outflux follows from the linearized continuity equation, neglecting cross-field electron fluxes and parallel ion flux: $\left.\partial_{t} n_{e}\right|_{j_{\|}}=-\nabla_{\|}\left(n_{e} u_{\| e}\right) \approx \nabla_{\|}\left(j_{\|} / e\right)$, or $\left.\partial_{t} n\right|_{j_{\|}} \approx \nabla_{\|}\left(j_{\|} / n_{e 0} e\right)$. Considering a single Fourier mode, with $k_{\perp}$ being the cross-field wave number, one finds that

$$
\frac{\left.\partial_{t} n\right|_{j_{\|}}}{\left.\partial_{t} \varphi\right|_{j_{\|}}} \approx-k_{\perp}^{2} \rho_{s}^{2}
$$

Parallel electron response and perpendicular ion polarization response combine to form a negative feedback loop. Although lack of parallel electron force balance always excites a parallel electron particle flux, this flux causes both density and potential changes through which it restores electron parallel force balance.

A few notes on Eq. (1). First, the result is totally insensitive to the manner in which the parallel electron current is determined. Whether the effective parallel impedance follows from collisional, electromagnetic, or inertial/kinetic effects is irrelevant to Eq. (1), which in fact depends only on the ion polarization physics. Second, the presence of $\rho_{s}$ does not indicate finite Larmor radius effects - the electron gyroradius is negligibly small because of the small electron mass, while the ion gyroradius is negligibly small because $T_{i}=0$. Rather, $\rho_{s}$ appears because of the balance between parallel electron response to $\varphi$, bringing in $T_{e}$, and ion polarization response, bringing in $m_{i}$. Third, in the frequently considered limit $k_{\perp} \rho_{s} \ll 1$, Eq. (1) takes the limit $\left.\partial_{t} n\right|_{j_{\|}} /\left.\partial_{t} \varphi\right|_{j_{\|}} \rightarrow 0$. This occurs because the ion polarization response becomes weak, so the change in the electric potential $\varphi$ must be relatively large to excite the quasineutral ion density response. As a result, the electron adiabatic response in the $k_{\perp} \rho_{s} \ll 1$ limit does not affect electron density at all, but rather controls the electrostatic potential, forcing $\varphi$ to remain equal to $n$. Intuitive understanding of drift wave physics is greatly clarified by a solid grasp of this simple fact.

Using the preceding results, consider now the physical mechanism of the "textbook" isothermal, resistive drift wave. Assume a simple slab geometry, with $\boldsymbol{B}=$ $B_{0} \hat{\boldsymbol{z}}$ and $\nabla n_{0}=-\left(n_{0} / L_{n}\right) \hat{\boldsymbol{x}}$, c.f. Fig. 2. Start with a sinusoidal adiabatic fluctuation $n(y, z)=\varphi(y, z)=$ $\hat{\varphi} \sin \left(k_{y} y\right) \cos \left(k_{\|} z\right)$. The $y$-derivative of $\varphi$ excites an $x$ directed $\boldsymbol{E} \times \boldsymbol{B}$ drift, $v_{E x}$, which causes a $\partial_{t} n$ due to the background density gradient. Since the $\boldsymbol{E} \times \boldsymbol{B}$ drift advects ions and electrons identically in the cold-ion limit, the potential is unchanged to this point. However, $v_{E x}$ and $\partial_{t} n$ are zero in the plane with $k_{\|} z=\pi / 2$ (out of the page), so the electrons are now out of parallel force balance. Electrons therefore flow quickly out of the density hump along the magnetic field, rapidly causing $\varphi$ and $n$ to become equal. For $k_{\perp} \rho_{s} \ll 1$, only $\varphi$ is nonnegligibly affected by the parallel electron flux, which ties it to $n$. Otherwise, the divergence of the parallel density flux is also non-negligible and opposes $\boldsymbol{v}_{E} \cdot \nabla n_{0}$. This slows the drift wave, causing the dispersive correction $1 /\left(1+k_{\perp}^{2} \rho_{s}^{2}\right)$

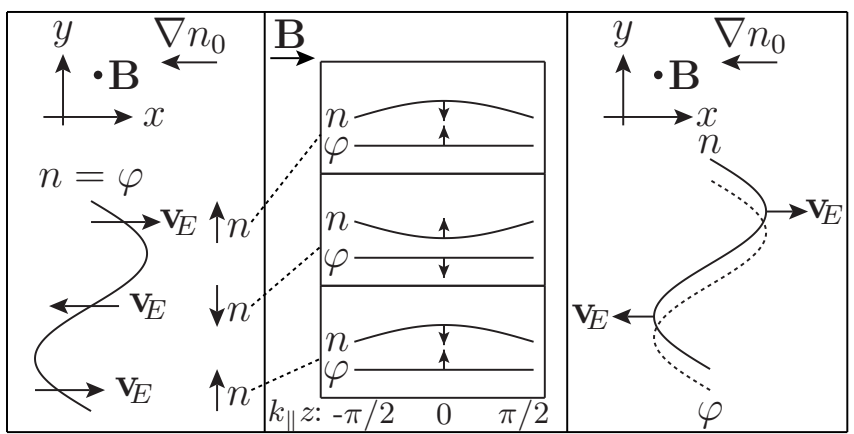

Figure 2. "Textbook" drift wave instability. Left: An initially adiabatic fluctuation [with horizontal deflection showing $n(y)=\varphi(y)$ ] excites an $\boldsymbol{E} \times \boldsymbol{B}$ drift up and down the gradient, causing a $\partial_{t} n$. Middle: The parallel electron response causes a corresponding $\partial_{t} \varphi$. Right: Nonvanishing parallel resistivity causes a phase shift between $n$ and $\varphi$ that leads to instability.

to the drift wave frequency. Assuming infinitely fast parallel electron response, $\varphi=n$ is maintained at all times, thus $\partial_{t} n \propto v_{E x} \propto \partial_{y} \varphi$ is poloidally $\pi / 2$ out of phase with $n$ at all times and the wave propagates upward (electron diamagnetic direction) without growing or damping.

Suppose instead that the parallel electron response were rapid but not infinitely so, a "near-adiabatic"case in which electrons were slightly slowed by electron-ion collisions. Considering again Fig. 2, this means that after $v_{E x}$ changes $n$, but not $\varphi$, a small but finite time lag is required before the parallel dynamics causes the corresponding $\partial_{t} \varphi$. Thus $n$ leads $\varphi$ slightly in time and, since the wave propagates upward, $n$ is shifted slightly upward from $\varphi$ in the diagram. This phase shift means that $v_{E x}$ is slightly outward at the maximum $n$ and inward at the most negative $n$, thus the drift wave is now growing in amplitude. This phase shift, due to the finite electron parallel response time, is at the heart of the drift wave instability.

As we will see, the parallel electron response may be fairly linear even when the perpendicular physics is strongly nonlinear. In fact, the basic physics of this section holds true for a wide variety of more general cases. In the regime of weak parallel impedance, this will allow the systematic derivation of an approximate one-field model, using a frequency ordering to effectively "slave" a $(\varphi-n)$-type variable to a $(\varphi=n)$-type variable. The resulting model catches the sensitive density-potential correlations, including the important contribution of the vorticity nonlinearity (physically due to the nonlinear polarization drift), in the functional form of the slaved variable, removing that burden from further analytic manipulations such as statistical closures.

\section{EQUATIONS AND VARIABLE TRANSFORMATION}

The HWEs analyzed here represent an extreme simplification of tokamak edge turbulence. They are local, elec- 
trostatic, resistive, isothermal equations in a slab geometry. Ions are assumed cold, eliminating FLR effects. Parallel ion flux and electron mass are neglected. Toroidal effects are missing, including coupling to geodesic acoustic modes, rendering the HWEs inappropriate for quantitative zonal flow studies. ${ }^{35,36}$ Nevertheless, the HWEs form a useful paradigm problem, due to their inclusion of nonlinearly coupled unstable and damped modes, selfconsistently determined linear and nonlinear drive, and the coupled $\boldsymbol{E} \times \boldsymbol{B}$ and vorticity nonlinearities.

Using the resistive Ohm's Law $C j_{\|}=\nabla_{\|}(n-\varphi)$, the HWEs may be written as

$$
\begin{gathered}
\partial_{t} n+\{\varphi, n\}+\omega_{n} \partial_{y} \varphi=\nabla_{\|} j_{\|}=C^{-1} \nabla_{\|}^{2}(n-\varphi), \\
\partial_{t} \nabla_{\perp}^{2} \varphi+\left\{\varphi, \nabla_{\perp}^{2} \varphi\right\}=\nabla_{\|} j_{\|}=C^{-1} \nabla_{\|}^{2}(n-\varphi) .
\end{gathered}
$$

Eq. (2a) advances fluctuations of density from a background mean value $n_{0}$, which is assumed constant with constant radial gradient $-n_{0} / L_{n}$, a radially local approximation. Fluctuating density $n$ is normalized to $n_{0}\left(\rho_{s} / L_{\perp}\right)$, potential $\varphi$ to $\left(T_{e 0} / e\right)\left(\rho_{s} / L_{\perp}\right)$, parallel current $j_{\|}$to $n_{0} e c_{s}\left(\rho_{s} / L_{\perp}\right)\left(L_{\|} / L_{\perp}\right)$, time $t$ to $L_{\perp} / c_{s}$, radial $x$ and binormal $y$ coordinates to $\rho_{s}$, and parallel coordinate $z$ to $L_{\|}$, with $L_{\|} \gg L_{\perp}$ of order the connection length. The standard normalization choice $L_{\perp}=L_{n}$ sets $\omega_{n} \doteq L_{\perp} / L_{n}$ to 1 . The parallel resistivity parameter $C \doteq 0.51\left(m_{e} / m_{i}\right)\left(\nu_{e i} L_{\perp} / c_{s}\right)\left(L_{\|}^{2} / L_{\perp}^{2}\right)$ is large in the hydrodynamic regime and small in the adiabatic regime. ${ }^{37}$ The Poisson-bracket notation $\{\varphi, n\} \doteq$ $\left(\partial_{x} \varphi\right)\left(\partial_{y} n\right)-\left(\partial_{y} \varphi\right)\left(\partial_{x} n\right)$ indicates the effects of $\boldsymbol{E} \times \boldsymbol{B}$ advection.

Unless otherwise noted, Eqs. (2) may be considered to be either $2 \mathrm{D}$ or $3 \mathrm{D}$. They are to be solved in a periodic domain of normalized dimensions $L_{x}, L_{y}$, and (for 3D) $L_{z}$. For $2 \mathrm{D}$ equations, substitute $\nabla_{\|}^{2} \rightarrow-k_{\|}^{2}$ for nonzonal components and $\nabla_{\|}^{2} \rightarrow 0$ for zonal components, with the constant $k_{\|}$nominally unity. ${ }^{38}$ Although wellmotivated by geometrical constraints, ${ }^{16}$ the $2 \mathrm{D}$ model of course misses modal resonances with rational surfaces as well as the nonlinear cascade in $k_{\|}$. For a realistic 3D model, some form of magnetic shear must be incorporated: The simplest shearless model, $\nabla_{\|} \rightarrow \partial_{z}$ with simply periodic boundary conditions in $z$, admits a $k_{\|}=0$ mode for each and every $\boldsymbol{k}_{\perp}$, which is grossly inconsistent with toroidal and poloidal periodicity for nonvanishing rotational transform.

As discussed in Sec. II, the relaxation towards electron adiabatic response is mediated by the parallel current. The decomposition of an arbitrary potential $\varphi$ into adiabatic $(a)$ and nonadiabatic $(b)$ portions may therefore be defined by a pair of requirements. First, $a$ must not appear under the parallel gradient in Ohm's Law. Second, $j_{\|}$must not appear in the evolution equation for $a$. [With such a definition, neglect of the $\boldsymbol{E} \times \boldsymbol{B}$ and nonlinear polarization drifts in Eqs. (2) must lead to a system that damps $\nabla_{\|} b$ to zero, leaving $a$ unaffected. The definition eliminates the participation of $a$ in the rapid parallel current response, much as the guiding-center coordinate transform removes the leading-order contribution of the rapid gyromotion.] To meet the first criterion, and thereby ensure that purely adiabatic fluctuations $\left(\nabla_{\|} a \neq 0, \nabla_{\|} b=0\right)$ not excite $j_{\|}$, one must set $b \propto \varphi-n$. In order that $j_{\|}$not appear in the evolution equation for $a$, the relative forms of Eqs. (2) force one to choose $a \propto n-\nabla_{\perp}^{2} \varphi$. (This form follows from the relative response of $n$ and $\varphi$ to $j_{\|}$which, as discussed in Sec. II, is entirely independent of the Ohm's Law, depending only on ion polarization physics.) Up to convenient scale-byscale normalizations, the new variables are thus uniquely specified as

$$
a \doteq\left(1-\nabla_{\perp}^{2}\right)^{-1}\left(n-\nabla_{\perp}^{2} \varphi\right), \quad b \doteq\left(1-\nabla_{\perp}^{2}\right)^{-1}(\varphi-n)
$$

In terms of $a$ and $b$, the original variables are simply $\varphi=a+b$ and $n=a+\nabla_{\perp}^{2} b$, showing that $a$ and $b$ correspond to the adiabatic and nonadiabatic portions of $\varphi$. The low- $k_{\perp}$ limits of these relations, $a \rightarrow n, b \rightarrow \varphi-n$, highlight the fact that the electron adiabatic response controls $\varphi$ at low $k_{\perp}$, no longer affecting $n$ (c.f. Sec. II). The inverse operator $\left(1-\nabla_{\perp}^{2}\right)^{-1}$ indicates a smoothing operation, dividing each Fourier component by $1+k_{\perp}^{2}$.

Although transformation of the HWEs to this variable pair appears to be new, ${ }^{39}$ each of the variables has been extensively used separately. Note, however, that the fluctuations represented by $a$ depend also on the definition of $b$, and vice versa, as for example alteration of the poloidal angle represents different real-space displacements in field-line-following and non-field-line-following coordinate systems. ${ }^{40}$ The combination $\ln n_{\text {tot }}-\nabla_{\perp}^{2} \varphi$, an inviscid Lagrangian invariant of the HWEs and similar plasma equations, has been used often. ${ }^{41}$ Helpfully for physical intuition, $a$ is scale-by-scale proportional to the cold-ion limit of the ion gyrocenter density used in gyrofluid models, ${ }^{15,42,43}$ a correspondence that follows from the fact that the polarization drift is absorbed into the coordinate transform from particle to gyrocenter position. ${ }^{44}$ The nonadiabatic electron density is extensively used in near-adiabatic approximations, ${ }^{25-27,31,32}$ as well as in other contexts. Note also that $a$ and $b$ are the amplitudes that result from projection onto the $C \rightarrow 0$ limit of the drift-wave $(\varphi=n)$ and damped $\left(n=\nabla_{\perp}^{2} \varphi\right)$ eigenmodes of the HWEs, respectively. There is thus a connection with nonlinear methods that project onto the eigenmodes, ${ }^{45}$ although in the present work we obtain exact equations for limiting eigenmodes rather than approximate equations for the exact eigenmodes. Also, as shown in upcoming publications, the $a$ and $b$ variables may be similarly employed in fuller systems for which they do not constitute the $C \rightarrow 0$ eigenmodes.

Upon linearly recombining Eqs. (2), expressing $n$ and $\varphi$ as functions of $a$ and $b$, simplifying using the bilinearity and antisymmetry of the Poisson bracket, and adding a positive dissipation operator $\mathcal{D}_{a}$ acting on $a,{ }^{46}$ one ob- 
tains

$$
\begin{array}{cc}
\partial_{t}\left(1-\nabla_{\perp}^{2}\right) a+\omega_{n} \partial_{y} \tilde{a}-\left\{a, \nabla_{\perp}^{2} a\right\}+\mathcal{D}_{a} a & \\
=-\omega_{n} \partial_{y} \tilde{b}-\left\{b,\left(1-\nabla_{\perp}^{2}\right) a\right\}, & (4 \mathrm{a}) \\
\partial_{t} \nabla_{\perp}^{2}\left(1-\nabla_{\perp}^{2}\right) b-\omega_{n} \partial_{y} \nabla_{\perp}^{2}(\tilde{a}+\tilde{b})+\left\{a+b, \nabla_{\perp}^{2}(a+b)\right\} \\
-\nabla_{\perp}^{2}\left\{a+b, a+\nabla_{\perp}^{2} b\right\}=\left(1-\nabla_{\perp}^{2}\right) \nabla_{\|} j_{\|}, & (4 \mathrm{~b}) \\
C j_{\|}=-\nabla_{\|}\left(1-\nabla_{\perp}^{2}\right) \tilde{b} . & (4 \mathrm{c})
\end{array}
$$

Except for $\mathcal{D}_{a}$, Eqs. (4) have content identical to Eqs. (2), simply expressed in the new variables. In particular, Eqs. (4) hold for all values of $C$. For later convenience, a decomposition into zonal $\bar{b} \doteq\left(L_{y} L_{z}\right)^{-1} \int d y \int d z b$ and and nonzonal $\tilde{b} \doteq b-\bar{b}$ components has been used. Dissipation acting on $a$ must be independent of $b$ in order to guarantee positive dissipation of the mean-squared fluctuating ion gyrocenter density $\mathcal{N}_{i}$ (c.f. Sec IV), as shown in Appendix A.

In the adiabatic $(b=0)$, nondissipative $\left(\mathcal{D}_{a}=0\right)$ limit, Eq. (4a) reduces to the HM equation, ${ }^{24}$ thus adiabatic self-dynamics reflect HM dynamics, including the dual cascade resulting from concurrent nonlinear conservation of energy and enstrophy. Drive, dissipation via $j_{\|}$, and the $\boldsymbol{E} \times \boldsymbol{B}$ nonlinearity (and resulting direct cascade) are explicitly proportional to $b$. As desired, the parallel current dissipates $b$ without appearing in the evolution equation for $a$. Also, although Eq. (4b) appears complicated, it leads to simple forms for the nonlinear invariants (Sec. IV) and the near-adiabatic approximation (Sec. V).

Of course, one may recapture the well-known linear modes of the HWEs from Eqs. (4), neglecting $\mathcal{D}_{a}$ and considering complex amplitudes $\hat{a}_{\boldsymbol{k}}$ and $\hat{b}_{\boldsymbol{k}}$ for wave vector $\boldsymbol{k}$. When $\omega_{\text {lin }} \doteq k_{y} \omega_{n} /\left(1+k_{\perp}^{2}\right)$ is much smaller than $\eta_{\mathrm{c}} \doteq k_{\|}^{2}\left(1+k_{\perp}^{2}\right) / C k_{\perp}^{2}$, one obtains two well-separated eigenmodes. The rapidly damped $\omega \approx-i \eta_{\mathrm{c}}$ resistive decay eigenmode is almost completely nonadiabatic, $\hat{a}_{\boldsymbol{k}} \approx$ $i\left(\omega_{\operatorname{lin}} / \eta_{\mathrm{c}}\right) \hat{b}_{\boldsymbol{k}} \ll \hat{b}_{\boldsymbol{k}}$, capturing the strong dissipation of nonadiabatic fluctuations by the parallel current. The low-frequency $\omega \approx \omega_{\text {lin }}$ drift wave eigenmode is nearly adiabatic, $\hat{b}_{\boldsymbol{k}} \approx i\left(\omega_{\text {lin }} / \eta_{\mathrm{c}}\right) \hat{a}_{\boldsymbol{k}} \ll \hat{a}_{\boldsymbol{k}}$, with correspondingly small growth rate $\gamma \approx \omega_{\text {lin }}\left(\omega_{\text {lin }} / \eta_{\mathrm{c}}\right)$. For $\omega \approx \omega_{\text {lin }}, b$ has a low enough amplitude that its only non-negligible contribution to its own linear equation occurs via the parallel current term $\propto C^{-1}$. In particular, one may discard the time derivative term, thus solving for $b$ as a function of $a$ rather than dynamically evolving it. Despite its smallness, $b$ remains important since its contribution to the $a$ equation determines the growth rate. This basic situation persists for the nonlinear problem, enabling the weakly-nonadiabatic approximation discussed in Sec. V.

When is the drift wave eigenmode weakly nonadiabatic? The resistive decay rate $\eta_{\mathrm{c}}$ grows as $k_{\perp}^{-2}$ for $k_{\perp}<1$, despite the fact that the response of $j_{\|}$to $(\varphi-n)$ is $k_{\perp}$-independent, simply because it is $\nabla_{\perp}^{2} \varphi$ (not $\varphi$ itself) that responds to $j_{\|}$. Since $\omega_{\text {lin }} \propto k_{y}$ at low $k_{\perp}$, the ratio $\omega_{\text {lin }} / \eta_{\mathrm{c}} \propto C k_{y} k_{\perp}^{2}$ drops rapidly with decreasing $k_{\perp}$. For experimental tokamak edge parameters, $C$ is often of order unity, thus $\omega_{\operatorname{lin}} / \eta_{\mathrm{c}}$ always becomes small as $k_{\perp}$ drops even modestly below unity, justifying the weakly nonadiabatic treatment of linear drift waves for those $k_{\perp}$. In contrast, the commonly considered "hydrodynamic limit" of the HWEs corresponds to setting $\eta_{\mathrm{c}}=0$, which is never justified for realistic tokamak edge parameters once $k_{\perp}$ drops to order $1 / 10$, well within the active turbulent spectrum.

\section{NONLINEAR INVARIANTS}

The existence of integral quantities conserved by the nonlinear terms is known to place important constraints on the evolution of a turbulent system. For example, in 2D neutral fluid turbulence, the simultaneous nonlinear conservation of both energy and enstrophy implies a dual cascade in which energy is transferred from smaller to larger spatial scales. ${ }^{47}$ In this section, implications of the nonlinearly-conserved quantities of the HWEs will be derived and discussed: Concurrent energy and $\mathcal{N}_{i}$ balance will yield a constraint linking the spectra of the $a$ and $b$ variables purely through the dissipation operators. Heuristic interpretation of the energy balance equations will provide intuition about the self-sustained turbulence exhibited by the HWEs. ${ }^{13}$ Energy balance arguments will lead to a rigorous upper bound on the ratio of nonadiabatic to adiabatic energy for nondecaying solutions.

The HWEs are known to possess the four nonlinear invariants $\frac{1}{2}\left\langle\left|\nabla_{\perp} \varphi\right|^{2}\right\rangle, \frac{1}{2}\left\langle\left(\nabla_{\perp}^{2} \varphi\right)^{2}\right\rangle, \frac{1}{2}\left\langle n^{2}\right\rangle$, and $\left\langle n \nabla_{\perp}^{2} \varphi\right\rangle$, in which angle brackets indicate spatial averaging over the domain. ${ }^{37}$ Of these four invariants, one may construct two linearly independent invariants for which the $j_{\|}$term may not act as a source: an energy $\mathcal{E} \doteq \frac{1}{2}\left\langle n^{2}+\left|\nabla_{\perp} \varphi\right|^{2}\right\rangle$ and the mean-squared fluctuating ion gyrocenter density $\mathcal{N}_{i} \doteq \frac{1}{2}\left\langle\left(n-\nabla_{\perp}^{2} \varphi\right)^{2}\right\rangle .{ }^{48}$ Using Eqs. (4) and some integrations by parts, one straightforwardly obtains

$$
\begin{aligned}
\partial_{t} \mathcal{E}_{a}= & -\omega_{n}\left\langle a \partial_{y} \tilde{b}\right\rangle-\left\langle b\left\{a, \nabla_{\perp}^{2} a\right\}\right\rangle-\left\langle a \mathcal{D}_{a} a\right\rangle, \\
\partial_{t} \mathcal{E}_{b}= & \omega_{n}\left\langle\left(\nabla_{\perp}^{2} a\right) \partial_{y} \tilde{b}\right\rangle+\left\langle b\left\{a, \nabla_{\perp}^{2} a\right\}\right\rangle \\
& -C^{-1}\left\langle\left[\nabla_{\|}\left(1-\nabla_{\perp}^{2}\right) \tilde{b}\right]^{2}\right\rangle, \\
\partial_{t} \mathcal{N}_{i}= & -\omega_{n}\left\langle\left(a-\nabla_{\perp}^{2} a\right) \partial_{y} \tilde{b}\right\rangle-\left\langle\left(a-\nabla_{\perp}^{2} a\right) \mathcal{D}_{a} a\right\rangle,
\end{aligned}
$$

in which $\mathcal{E}_{a} \doteq \frac{1}{2}\left\langle a^{2}+\left|\nabla_{\perp} a\right|^{2}\right\rangle, \mathcal{E}_{b} \doteq \frac{1}{2}\left\langle\left|\nabla_{\perp} b\right|^{2}+\left(\nabla_{\perp}^{2} b\right)^{2}\right\rangle$, $\mathcal{E}=\mathcal{E}_{a}+\mathcal{E}_{b}$, and $\mathcal{N}_{i}=\frac{1}{2}\left\langle\left(a-\nabla_{\perp}^{2} a\right)^{2}\right\rangle \cdot{ }^{49}$ In the purely adiabatic limit, $b=0, \mathcal{E}$ and $\mathcal{N}_{i}-\mathcal{E}$ become the HasegawaMima energy and enstrophy, respectively. ${ }^{37}$ [Interestingly, although $a$ contributes to two invariants with differing powers of $k_{\perp}$, suggesting dual-cascade dynamics, $b$ contributes to only one, suggesting a direct cascade. Indeed, the $\boldsymbol{E} \times \boldsymbol{B}$ nonlinearity, active only for $b \neq 0$, transfers density fluctuations to small scales, acting concurrently with the vorticity nonlinearity and the linear parallel coupling of $n$ and $\varphi$ to determine the turbulent state. ${ }^{50,51}$ ] The $\omega_{n}$ terms represent the only 
source, density flux down the density gradient. Since such a flux requires nonadiabatic electrons with $y$ variation, these terms vanish for $\tilde{b}=0$. The parallel current $\left(C^{-1}\right)$ term represents definite dissipation, acting only on $\mathcal{E}_{b}$. The resulting dissipation rate becomes very rapid at low $k_{\perp}$, acting with a strength $\propto\left|\hat{b}_{\boldsymbol{k}}\right|^{2}$ on an energy $\propto k_{\perp}^{2}\left|\hat{b}_{\boldsymbol{k}}\right|^{2}$. The $\mathcal{D}_{a}$ term represents the comparatively weak direct dissipation on adiabatic fluctuations, probably dominated by ion Landau damping at small $k_{\perp}$ and ion-ion collisions (viscosity) at large $k_{\perp}$. The $\pm\left\langle b\left\{a, \nabla_{\perp}^{2} a\right\}\right\rangle$ terms represent energy exchange between adiabatic and nonadiabatic fluctuations due to the vorticity nonlinearity.

The $\mathcal{E}$ and $\mathcal{N}_{i}$ invariants have identical source terms but significantly different dissipation. Evaluating the evolution of $\mathcal{N}_{i}-\mathcal{E}$, one obtains

$$
\partial_{t}\left(\mathcal{N}_{i}-\mathcal{E}\right)=C^{-1}\left\langle\left[\nabla_{\|}\left(1-\nabla_{\perp}^{2}\right) \tilde{b}\right]^{2}\right\rangle-\left\langle\left(-\nabla_{\perp}^{2} a\right) \mathcal{D}_{a} a\right\rangle,
$$

in which $\mathcal{N}_{i}-\mathcal{E}=\frac{1}{2}\left\langle\left|\nabla_{\perp} a\right|^{2}+\left(\nabla_{\perp}^{2} a\right)^{2}-\left|\nabla_{\perp} b\right|^{2}-\left(\nabla_{\perp}^{2} b\right)^{2}\right\rangle$, an integral constraint on the steady-state spectra determined solely by the dissipation operators. Although the parallel current term $\propto C^{-1}$ represents by far the strongest dissipation operator for realistic parameter values, Eq. (6) implies that nonzero $\mathcal{D}_{a}$ is required for a steady-state solution. Additionally, a must either be of larger amplitude or have a broader spectrum than $b$ in order to achieve a steady state. Furthermore, let $k_{\perp}^{d} \doteq\left[\left\langle\left(-\nabla_{\perp}^{2} a\right) \mathcal{D}_{a} a\right\rangle /\left\langle a \mathcal{D}_{a} a\right\rangle\right]^{1 / 2}$ represent a characteristic $k_{\perp}$ for the adiabatic dissipation. The steadystate balance $\partial_{t}\left(\mathcal{N}_{i}-\mathcal{E}\right)=0$ then directly implies that $C^{-1}\left\langle\left[\nabla_{\|}\left(1-\nabla_{\perp}^{2}\right) \tilde{b}\right]^{2}\right\rangle \gg\left\langle a \mathcal{D}_{a} a\right\rangle$ if and only if $k_{\perp}^{d} \gg 1$, roughly, the parallel current dominates the energy sink if and only if adiabatic dissipation occurs mostly at large $k_{\perp}$. Conversely, $\partial_{t}\left(\mathcal{N}_{i}-\mathcal{E}\right)=0$ implies that direct adiabatic dissipation is the dominant energy sink if and only if $k_{\perp}^{d} \ll 1$. [In interpreting this, note that $\mathcal{N}_{i}$ may undergo a direct cascade, even if $\mathcal{E}$ has an inverse cascade, due to the presence of differing powers of $k_{\perp}$.]

The form of Eqs. (5a) and (5b) may aid intuitive understanding of self-sustained turbulence, sometimes also referred to as the nonlinear drift wave instability or submarginal turbulence. Numerical investigations of a superset of the HWEs have highlighted the role of the vorticity nonlinearity, which was found to cause self-sustained turbulence by broadening the phase shifts between $\tilde{n}$ and $\tilde{\phi}$, that is, via nonlinear excitation of nonadiabatic fluctuations. ${ }^{11,13}$ In Eqs. (5a) and (5b), the $\pm\left\langle b\left\{a, \nabla_{\perp}^{2} a\right\}\right\rangle$ terms, both resulting from the vorticity nonlinearity, provide the only net $\mathcal{E}_{a} \leftrightarrow \mathcal{E}_{b}$ energy transfer mechanism allowing adiabatic fluctuations to excite nonadiabatic ones. Since $\tilde{b}$ is much more strongly damped than $a$ is, and since the energy source for $b$ is smaller at the relatively small $k_{\perp}$ for which the energy source typically peaks, one expects net energy transfer from $a$ to $b$. One may then ask whether $b$ obtains energy primarily from its source term $\left[\omega_{n}\left\langle\left(\nabla_{\perp}^{2} a\right) \partial_{y} \tilde{b}\right\rangle\right]$ or via transfer from $a\left[\left\langle b\left\{a, \nabla_{\perp}^{2} a\right\}\right\rangle\right]$, due respectively to the linear $\boldsymbol{E} \times \boldsymbol{B}$ drift and the vorticity nonlinearity (nonlinear polarization drift). In the first case, linear physics supports the $n-\varphi$ phase shift and the energy source, corresponding to standard linear instability drive. In the second case, $b$ (and therefore the energy source) are supported by nonlinear interactions, in particular the vorticity nonlinearity, potentially allowing a state of sustained turbulence even in the absence of a linear instability. Note that if the energy source is dominated by $k_{\perp}$ rather less than 1 and the energy sink is dominated by the parallel current channel, then the steady-state $\mathcal{E}$, $\mathcal{E}_{a}$, and $\mathcal{E}_{b}$ balances imply that $b$ must dominantly obtain energy via nonlinear transfer from $a$. Note also that the nonlinear term may transfer energy from higher- $k_{\perp} a$ to lower- $k_{\perp} b$, enhancing the wave number factors relative to those of the linear term at lower $k_{\perp}$. Also, due to the powers of $k_{\perp}$ in $\mathcal{E}_{b}$, transfer of energy into low- $k_{\perp} b$ may lead to relatively large $b$ amplitudes.

The form of Eqs. (5a) and (5b) allows one to rigorously bound the gradients of $\tilde{b}$ relative to those of $\tilde{a}$ for nondecaying 2D HW turbulence. Letting $k_{\|}$be a fixed constant (nominally 1$), \partial_{t} \mathcal{E} \geq 0$ directly implies $\omega_{n}\left\langle\left(\partial_{y} \tilde{a}\right)\left(1-\nabla_{\perp}^{2}\right) \tilde{b}\right\rangle \geq k_{\|}^{2} C^{-1}\left\langle\left[\left(1-\nabla_{\perp}^{2}\right) \tilde{b}\right]^{2}\right\rangle$. The Cauchy-Schwarz inequality implies $\left|\left\langle\left(\partial_{y} \tilde{a}\right)\left(1-\nabla_{\perp}^{2}\right) \tilde{b}\right\rangle\right| \leq$ $\left\langle\left(\partial_{y} \tilde{a}\right)^{2}\right\rangle^{1 / 2}\left\langle\left[\left(1-\nabla_{\perp}^{2}\right) \tilde{b}\right]^{2}\right\rangle^{1 / 2}$, allowing one to conclude that

$$
\left\langle\left[\left(1-\nabla_{\perp}^{2}\right) \tilde{b}\right]^{2}\right\rangle^{1 / 2} \leq C \omega_{n} k_{\|}^{-2}\left\langle\left(\partial_{y} \tilde{a}\right)^{2}\right\rangle^{1 / 2}
$$

for nondecaying 2D HW turbulence. If $\partial_{t} \mathcal{E} \geq 0$ holds only under some temporal or ensemble averaging, then Eq. (7) holds under the same averaging. In a $3 \mathrm{D}$ system, Eq. (7) holds with the substitution $k_{\|} \rightarrow k_{\| \text {,avg }} \doteq$ $\left\langle\left[\nabla_{\|}\left(1-\nabla_{\perp}^{2}\right) \tilde{b}\right]^{2}\right\rangle^{1 / 2} /\left\langle\left[\left(1-\nabla_{\perp}^{2}\right) \tilde{b}\right]^{2}\right\rangle^{1 / 2}$. Analytical and numerical studies show that the nonlinearity prevents the energy from concentrating in only extremely small $k_{\|}$ modes, ${ }^{17}$ suggesting that $k_{\| \text {,avg }}$ does not become small in practice. Note that since $\left\langle\left(\partial_{y} \tilde{a}\right)^{2}\right\rangle \leq\left\langle\left|\nabla_{\perp} \tilde{a}\right|^{2}\right\rangle \leq$ $\left\langle\tilde{a}^{2}+\left|\nabla_{\perp} \tilde{a}\right|^{2}\right\rangle$ and $\left\langle\left[\left(1-\nabla_{\perp}^{2}\right) \tilde{b}\right]^{2}\right\rangle \geq\left\langle\left|\nabla_{\perp} \tilde{b}\right|^{2}+\left(\nabla_{\perp}^{2} \tilde{b}\right)^{2}\right\rangle$, Eq. (7) implies an analogous relation for the nonzonal portions of $\mathcal{E}_{b}$ and $\mathcal{E}_{a}$. [If one retains $\mathcal{D}_{a}$, the relative amplitude $\left\langle\tilde{b}^{2}\right\rangle /\left\langle\tilde{a}^{2}\right\rangle$ may also be bounded, ${ }^{39}$ but this is unnecessary since only perpendicular gradients contribute to the non- $j_{\|}$dynamics of Eqs. (4).]

\section{NEAR-ADIABATIC APPROXIMATION}

When fluctuations are nearly but not totally adiabatic, the reduction from a two- to a one-field model provides a strong incentive to develop approximation schemes for the nonadiabatic response. The strongly nonlinear character of edge turbulence, exemplified by the nonlinear instability, suggests that one must go beyond linear $i \delta$ type approximations, in particular incorporating the contribution of the vorticity nonlinearity. While Crotinger and Dupree ${ }^{31}$ and Naulin and Spatschek ${ }^{32}$ have already developed schemes of this type, the formulation in the 
$a, b$ variables that is developed in this section is significantly simpler and physically more transparent. The resulting approximation holds for strongly nonlinear turbulence and approximately captures all energy source and sink terms. Physically, the scheme applies when the effects of parallel current driven by $\tilde{b}$ dominate those of the cross-field drifts due to $\tilde{b}$. Technically, the scheme will be worked out in 2D as a straightforward perturbation expansion around the purely-adiabatic limit $\tilde{b}=0$. Contributions of zonal $\bar{b}$ will be neglected here for simplicity, but are retained in Appendix B.

The purely adiabatic limit may be obtained simply by setting $C=0$ in Eqs. (4) while requiring frequencies and amplitudes to remain finite. Equation (4c) immediately implies $\tilde{b}=0$, thus the RHS of Eq. (4a) vanishes. The parallel current $j_{\|}$is determined by the nonzonal, zerothorder Eq. (4b),

$$
\left(1-\nabla_{\perp}^{2}\right) \nabla_{\|} j_{\|}=-\omega_{n} \partial_{y} \nabla_{\perp}^{2} \tilde{a}+\left\{\widetilde{a, \nabla_{\perp}^{2} a} .\right.
$$

Physically, Eq. (8) is the weak-impedance limit of the negative-feedback loop of Sec. II: when parallel impedance is very small, any perpendicular fluxes disturbing the adiabatic balance [RHS of Eq. (8): linear density flux $\boldsymbol{v}_{E} \cdot \nabla n_{0}$ and the nonlinear polarization current (vorticity nonlinearity)] immediately drive a parallel current such that the nonadiabatic fluctuations remain negligibly small. One has effectively ordered out the high-frequency resistive-decay mode, while retaining its effects on the low-frequency dynamics. [This solution procedure closely resembles the use of the quasineutrality equation to solve for $\varphi$, in which the change of the actual charge density is ordered out in favor of the plasma polarization response that it excites.] Notably, due to the definition of $a$, the parallel current is a slaved variable to zeroth order, dependent on $a$ but making no contribution to the evolution of $a$.

For the near-adiabatic case, allow $C$ to be nonzero but small while taking frequencies, amplitudes, and wavenumbers to be order unity. ${ }^{52}$ Equation (4c) then implies $\tilde{b} / j_{\|} \sim O(C) \ll 1$, so the (nonzonal) leadingorder balance of Eq. (4b) remains Eq. (8), implying $a / j_{\|} \sim O(1)$ thus $\tilde{b} / a \sim O(C)$. Thanks to the definition of $a$, only $\tilde{b}$ (and not $j_{\|}$itself) appears in Eq. (4a), so only the leading-order $j_{\|}$is required for first-order accuracy in evolving $a$, the only remaining dynamical variable. Combining Eqs. (4c) and (8), one obtains the weakly nonadiabatic formula $\tilde{b} \approx \tilde{b}_{\mathrm{n}}$ for

$\tilde{b}_{\mathrm{n}} \doteq C\left(1-\nabla_{\perp}^{2}\right)^{-1} \nabla_{\|}^{-2}\left(1-\nabla_{\perp}^{2}\right)^{-1}\left(\omega_{n} \partial_{y} \nabla_{\perp}^{2} \tilde{a}-\left\{\widetilde{a, \nabla_{\perp}^{2} a}\right\}\right)$

in which $\left(1-\nabla_{\perp}^{2}\right)^{-1}$ is a linear smoothing operation, corresponding to division of each Fourier component by $\left(1+k_{\perp}^{2}\right)$. Since we are considering the $2 \mathrm{D}$ HWEs, $\nabla_{\|}^{-2}$ corresponds to dividing by a fixed constant $-k_{\|}^{2}$, which is nominally $-1 .{ }^{53}$ Substituting the RHS of Eq. (9) for $b$ in Eq. (4a), one obtains the weakly nonadiabatic one-field approximation of the HW equations (in the absence of zonal $b$ )

$$
\begin{gathered}
\partial_{t}\left(1-\nabla_{\perp}^{2}\right) a+\omega_{n} \partial_{y} \tilde{a}-\left\{a, \nabla_{\perp}^{2} a\right\}+\widetilde{\mathcal{D}_{a} a} \\
\approx-C \omega_{n} \partial_{y} \frac{\omega_{n} \partial_{y} \nabla_{\perp}^{2} \tilde{a}-\left\{\widehat{\left.a, \nabla_{\perp}^{2} a\right\}}\right.}{\left(1-\nabla_{\perp}^{2}\right) \nabla_{\|}^{2}\left(1-\nabla_{\perp}^{2}\right)} \\
+\left\{\left(1-\nabla_{\perp}^{2}\right) a, C \frac{\omega_{n} \partial_{y} \nabla_{\perp}^{2} \tilde{a}-\left\{\widetilde{\left.a, \nabla_{\perp}^{2} a\right\}}\right.}{\left(1-\nabla_{\perp}^{2}\right) \nabla_{\|}^{2}\left(1-\nabla_{\perp}^{2}\right)}\right\} .
\end{gathered}
$$

Equations (9) and (10) again represent the weakimpedance limit of the negative-feedback loop of Sec. II, as discussed after Eq. (8). In this case, the time-partial and cross-field drift terms due to $\tilde{b}$ are nonzero but remain negligible relative to the parallel current term in Eq. (4b), which therefore again determines the slaved zeroth-order $j_{\|}$via Eq. (8), allowing the leading-order $\tilde{b}$ to be determined by Eq. (4c) after the fact. Although the resulting terms on the RHS of Eq. (10) are small $\sim O(C)$, they are important because they control the energy and $\mathcal{N}_{i}$ sources and the resistive energy sink [cf. Eqs. (5)].

Several comments on Eq. (10) and its derivation are in order. First, despite its simple form, Eq. (10) has retained the vorticity nonlinearity on an equal footing with the linear drift term, thus may be applied to strong turbulence whenever $C$ is small enough. This is important since plasma edge turbulence is typically in the stronglynonlinear regime, ${ }^{10,13}$ in fact even forced HM turbulence is strong for $k_{\perp}$ away from 1 , at least in the limit of large Reynolds number. ${ }^{54}$ Further, Eq. (10) retains the dispersive polarization corrections, which are seen to play an important role in edge turbulence even when the main energy source occurs for $k_{\perp} \sim 0.1 .^{15}$

Second, besides weak parallel impedance, this approximation scheme relies only on the forms of the parallel gradient terms in Ohm's Law and the relative responses of density and vorticity to $j_{\|}$, thus it is easily generalizable to more complex systems that retain these or similar forms, as will be presented in upcoming work. Physically, strongly-nonlinear cross-field drifts disturb adiabatic balance, while predominantly linear parallel physics returns the plasma towards adiabatic response. The present scheme exploits the nearly linear parallel response.

Third, the definitions of $a$ and $b$ are the reason for the simple form of Eqs. (9) and (10). Specifically, $b \propto \varphi-n$ allows an expansion in small amplitude, rather than a small phase shift. [If $b$ were not proportional to $\varphi-n$, Eq. (4c) would constrain some linear combination of $\tilde{a}$ and $\tilde{b}$ to be small, rather than $\tilde{b}$ itself.] Also, if $a$ had been alternatively defined not proportional to $n-\nabla_{\perp}^{2} \varphi$, then its evolution equation would contain an explicit $j_{\|}$, so the fact that $j_{\|} / a \sim O(1)$ would imply that a firstorder $j_{\|}$were necessary in order to obtain a first-order $a$. Since $\tilde{b} / j_{\|} \sim O\left(C^{1}\right)$, the $\tilde{b}$ terms in Eq. (4b) would need to be retained, including $\partial_{t} \tilde{b}$, necessitating an iterative approximation. 
The advantage of our definition of $a$ may be clearly seen via comparison of the present scheme with those of $\mathrm{CD}$ and NS. If one neglects perpendicular dissipation, the schemes of CD and NS are in fact identical. They effectively begin with equations fully equivalent to the $2 \mathrm{D}$ form of our Eqs. (2), ${ }^{55}$ except that they retain the parallel current damping on all modes including the zonal component. They expand an equation for $n-\nabla_{\perp}^{2} \varphi$ in strong parallel damping, using a leading-order approximation for $h_{e} \doteq n-\varphi$, resembling our approach. However, they then use $\varphi$ rather than $a$ as their remaining dynamical variable, necessitating a substitution for $\partial_{t} \varphi$ on their RHS, which they obtain via iteration using the zeroth-order LHS. Again neglecting perpendicular dissipation, NS and CD arrive at the same approximate form, which may be written in our notation (after simplification) as

$$
\begin{gathered}
\partial_{t}\left(1-\nabla_{\perp}^{2}\right) \varphi+\omega_{n} \partial_{y} \varphi-\left\{\varphi, \nabla_{\perp}^{2} \varphi\right\}=-C k_{\|}^{-2}\left(1-\nabla_{\perp}^{2}\right)^{-1} \times \\
{\left[-\left(1-\nabla_{\perp}^{2}\right)^{-1} \omega_{n} \partial_{y} \nabla_{\perp}^{2}\left(\omega_{n} \partial_{y} \varphi-\left\{\varphi, \nabla_{\perp}^{2} \varphi\right\}\right)\right.} \\
+\left\{\nabla_{\perp}^{2} \varphi,-\left(1-\nabla_{\perp}^{2}\right)^{-1}\left(\omega_{n} \partial_{y} \varphi-\left\{\varphi, \nabla_{\perp}^{2} \varphi\right\}\right)\right\} \\
\quad+\left\{\varphi, \nabla_{\perp}^{2}\left(1-\nabla_{\perp}^{2}\right)^{-1}\left(\omega_{n} \partial_{y} \varphi-\left\{\varphi, \nabla_{\perp}^{2} \varphi\right\}\right)\right\} \\
\left.+\left(1-\nabla_{\perp}^{2}\right)\left\{\varphi,\left(1-\nabla_{\perp}^{2}\right)^{-1}\left(\omega_{n} \partial_{y} \nabla_{\perp}^{2} \varphi-\left\{\varphi, \nabla_{\perp}^{2} \varphi\right\}\right)\right\}\right] .
\end{gathered}
$$

Comparison of Eq. (11) with the $\mathcal{D}_{a} \rightarrow 0$ limit of our Eq. (10), which is equivalent to $O\left(C^{1}\right)$, highlights the efficiency of the $a, b$ variables for this problem.

Consider now the appearance of the nonlinear invariants in the weakly nonadiabatic approximation. Identical manipulations to those of Sec. IV may be applied to Eq. (10) to obtain

$$
\begin{aligned}
\partial_{t} \mathcal{E}_{a} & =-\omega_{n}\left\langle a \partial_{y} \tilde{b}_{\mathrm{n}}\right\rangle-\left\langle\tilde{b}_{\mathrm{n}}\left\{a, \nabla_{\perp}^{2} a\right\}\right\rangle-\left\langle a \mathcal{D}_{a} a\right\rangle, \\
\partial_{t} \mathcal{N}_{i} & =-\omega_{n}\left\langle\left(a-\nabla_{\perp}^{2} a\right) \partial_{y} \tilde{b}_{\mathrm{n}}\right\rangle-\left\langle\left(a-\nabla_{\perp}^{2} a\right) \mathcal{D}_{a} a\right\rangle .
\end{aligned}
$$

The evolution equation for $\mathcal{E}_{b}$ has been replaced in the weakly nonadiabatic approximation by the easily verified relation

$$
\begin{aligned}
& C^{-1}\left\langle\left[\nabla_{\|}\left(1-\nabla_{\perp}^{2}\right) \tilde{b}_{\mathrm{n}}\right]^{2}\right\rangle \\
&=-C^{-1}\left\langle\tilde{b}_{\mathrm{n}}\left(1-\nabla_{\perp}^{2}\right) \nabla_{\|}^{2}\left(1-\nabla_{\perp}^{2}\right) \tilde{b}_{\mathrm{n}}\right\rangle \\
& \quad=\omega_{n}\left\langle\left(\nabla_{\perp}^{2} a\right) \partial_{y} \tilde{b}_{\mathrm{n}}\right\rangle+\left\langle\tilde{b}_{\mathrm{n}}\left\{a, \nabla_{\perp}^{2} a\right\}\right\rangle .
\end{aligned}
$$

Comparing with Eq. (5b), one sees that $\tilde{b}_{\mathrm{n}}$ has been chosen just such that the total energy contributions to $\partial_{t} \mathcal{E}_{b}$ sum to zero. One may substitute this result into $\mathcal{E}_{a}$ to obtain the alternate form

$$
\begin{aligned}
\partial_{t} \mathcal{E}_{a}=-\omega_{n}\left\langle\left(a-\nabla_{\perp}^{2} a\right) \partial_{y} \tilde{b}_{\mathrm{n}}\right\rangle-\left\langle a \mathcal{D}_{a} a\right\rangle \\
-C^{-1}\left\langle\left[\nabla_{\|}\left(1-\nabla_{\perp}^{2}\right) \tilde{b}_{\mathrm{n}}\right]^{2}\right\rangle,
\end{aligned}
$$

just the equation for $\mathcal{E}$ with $b \rightarrow \tilde{b}_{\mathrm{n}}$. The approximation is thus a simplification of the original energy balance with $b \rightarrow \tilde{b}_{\mathrm{n}}$ and $\mathcal{E}_{b} \rightarrow 0$.
Despite the close similarity, it should be stressed that the nonlinear term in $\tilde{b}_{\mathrm{n}}$ implies that nonlinear terms now appear in both source and sink roles for $\mathcal{E}_{a}$ and $\mathcal{N}_{i}$. This nonlinear term appears in both the $C^{-1}$ term, which represents a definite sink just as in Eqs. (5), and in the source terms $\propto \omega_{n}$, which again are of indefinite sign in general, but must be positive for a turbulent steady state. Note also that the nonlinear portion of $\tilde{b}_{\mathrm{n}}$ contributes a cubic nonlinearity to Eq. (10), which represents a definite $\operatorname{sink}$ for $\mathcal{E}_{a}$ but does not contribute to $\mathcal{N}_{i}$.

The derivation of Eqs. (9) and (10) took $k_{\perp} \sim O(1)$ while $C \ll 1$, but one would like the resulting equations to approximately hold over all relevant $k_{\perp}$. In particular, given the well-known inverse cascade in the purely adiabatic limit, do the terms retained in Eq. (8) continue to dominate the other terms of Eq. (4b) as $k_{\perp}$ gets small? To estimate, one may compare each neglected term with the $j_{\|}$term, using Eq. (4c) to relate the Fourier amplitudes $\left|\hat{b}_{\boldsymbol{k}}\right| /\left|\hat{j}_{\| \boldsymbol{k}}\right|=C / k_{\|}\left(1+k_{\perp}^{2}\right)$. Assuming that interactions are local in $k_{\perp}$, as seen in simulations, ${ }^{51}$ and estimating the $k_{\perp}$-dependent frequency content $\omega\left(k_{\perp}\right) \sim$ $\max \left[k_{y} \omega_{n}, k_{\perp}^{4} a_{k_{\perp}}\right] /\left(1+k_{\perp}^{2}\right)$, the neglected terms drop faster than the $j_{\|}$term for decreasing $k_{\perp}<1$, as long as $a_{k_{\perp}}$ grows more slowly than $k_{\perp}^{-4} .{ }^{56} \mathrm{~A}$ simple Kolmogorov analysis ${ }^{54}$ of Eq. (4a) neglecting $b$ and $\mathcal{D}_{a}$ suggests that $a_{k_{\perp}} \propto k_{\perp}^{-4 / 3}$ for $k_{\perp} \ll 1$, well satisfying the required bound, in fact further suggesting that lower- $k_{\perp}$ portions of the spectrum may typically be weakly nonadiabatic even when $C$ is order unity. Since the relative magnitude of the neglected terms does not grow at large $k_{\perp}$ if $a_{k_{\perp}}$ drops off at least as $k_{\perp}^{-2}$, which is predicted by a Kolmogorov-like analysis of the HM enstrophy cascade for $k_{\perp}>1,{ }^{54}$ convergence of the approximation for $k_{\perp} \sim 1$ suggests that the approximation should converge for all $k_{\perp}$.

One may analytically estimate the dominant error in $\tilde{b}_{\mathrm{n}}$ for the case of purely nonzonal fluctuations by taking the difference of the nonzonal portions of Eqs. (4b) and (8), approximating $\partial_{t} \nabla_{\perp}^{2}\left(1-\nabla_{\perp}^{2}\right) \tilde{b}$ using Eq. (9) and the zeroth-order Eq. (4a), ${ }^{57}$ simplifying the result using $\tilde{b} \approx \tilde{b}_{\mathrm{n}}$, and assuming the spectrum of $\tilde{a}$ is concentrated at much lower $k_{\perp}$ than that of $\tilde{b}$, obtaining the $2 \mathrm{D}$ relation $\tilde{b}_{\mathrm{n}}-\tilde{b}=2 C k_{\|}^{-2}\left(1-\nabla_{\perp}^{2}\right)^{-2}\left[\omega_{n} \partial_{y} \nabla_{\perp}^{2} \tilde{b}-\right.$ $\left.\left\{\widetilde{a, \nabla_{\perp}^{2}} b\right\}+\cdots\right]$, in which omitted terms are either higher order in $\tilde{b} / \tilde{a}$ or involve higher-order derivatives acting on $a$. The corresponding simple estimate $\left\langle\left(\tilde{b}_{\mathrm{n}}-\right.\right.$ $\left.\tilde{b})^{2}\right\rangle \sim\left\langle\left(\tilde{b}_{\mathrm{n}}-\tilde{b}\right)^{2}\right\rangle_{\text {est }} \doteq 4 C^{2} k_{\|}^{-4}\left[\omega_{n}^{2}\left\langle\left|\nabla_{\perp}^{3} \tilde{b} /\left(1-\nabla_{\perp}^{2}\right)^{2}\right|^{2}\right\rangle+\right.$ $\left.\left\langle\left|\nabla_{\perp} a\right|^{2}\right\rangle\left\langle\left|\nabla_{\perp}^{3} \tilde{b} /\left(1-\nabla_{\perp}^{2}\right)^{2}\right|^{2}\right\rangle\right]$ indeed tracks $\left\langle\left(\tilde{b}_{\mathrm{n}}-\tilde{b}\right)^{2}\right\rangle$ reasonably well in numerical simulations, as shown in Fig. 4.

\section{NUMERICAL VERIFICATION}

Equation (9) provides an approximate formula $\tilde{b}_{\mathrm{n}}(a)$ for $\tilde{b}$, expected to hold in the limit of small normalized parallel resistivity $C$. The principal underlying ordering, 
$\tilde{b} / \tilde{a} \sim O(C)$, was established rigorously for nondecaying turbulence using energy arguments [Eq. (7)]. However, this does not conclusively prove that all terms neglected from Eq. (4b) to get Eq. (8) are negligible at small but finite $C$, primarily because of the varying powers of $k_{\perp}$. While scaling arguments near the end of Sec. V suggest that the approximation in fact converges over the whole spectrum, direct verification of this convergence is desirable. This section provides such verification by numerically solving Eqs. (2) in 2D, post-processing the results to evaluate $a$ and $b$, and comparing the prediction $\tilde{b}_{\mathrm{n}}(a)$ with the actual $\tilde{b}$. An $i \delta$-like linear prediction $\tilde{b}_{\ell}$, defined to be the linear term of $\tilde{b}_{\mathrm{n}}$, is found to incur order-unity errors for all $C$.

Equations (2) were numerically solved with periodic boundary conditions using a modification of the 2D code of Ref. 58. ${ }^{59}$ Poisson brackets and $\omega_{n} \partial_{y} \varphi$ were evaluated using a 4 th-order Arakawa scheme, ${ }^{60}$ while all other spatial derivatives were evaluated spectrally. A 3rd-order Karniadakis scheme ${ }^{61}$ was used for time-stepping, treating parallel conduction implicitly and all other terms explicitly. Grid-scale dissipation was introduced with explicit hyperviscous damping, adding terms $-\nu \nabla_{\perp}^{4} n$ and $-\nu \nabla_{\perp}^{4}\left(\nabla_{\perp}^{2} \varphi\right)$ to the RHS of Eqs. (2a) and (2b), respectively. (This dissipation scheme represents positivedefinite dissipation for both $\mathcal{E}$ and $\mathcal{N}_{i}$, as discussed in Appendix A.) Zonal components were zeroed out for simplicity. $C$ was scanned from 10 down to $10^{-2}$, with nominal parameters $\omega_{n}=1$ and $\nabla_{\|}^{2}=-1$. The simulation domain was $80 \pi \rho_{s} \times 80 \pi \rho_{s}$, with a resolution-refining procedure used to efficiently obtain convergence, as detailed in Appendix C. Simulations were initialized with a randomly-phased order-unity bath of adiabatic fluctuations, with $\left\langle n^{2}\right\rangle k_{\perp}$-spectrum $\propto k_{\perp} /\left[1+\left(k_{\perp} / k_{\perp 0}\right)^{8}\right]$ for $k_{\perp 0} \approx 0.28$.

The steady-state spectra of $n, \varphi$, and $\left|\nabla_{\perp} \varphi\right|$ all exhibit a central peak in $k_{\perp}$, dropping for both smaller and larger wave numbers. As $C$ decreases, the location of the peak shifts to lower $k_{\perp}$, from near 1 for $C=10$ to a little below 0.1 for $C=0.01$. However, $k_{\perp} \approx 1$ dynamics contribute significantly to the energy source and sink terms at all $C$, since nonadiabatic fluctuations remain strongest there even at small $C$. Spectral evolution slows down as $C$ decreases, with only $\sim O\left(10^{2}\right)$ normalized times required for $C=10$ and 1 cases to saturate, but $\sim O\left(10^{5}\right)$ for $C=0.1$ and $\gtrsim O\left(10^{7}\right)$ for $C=0.01$.

The numerical results, drawn from periods of saturated steady-state turbulence, ${ }^{62}$ confirm that the nonlinear estimate $\tilde{b}_{\mathrm{n}}$ does indeed converge to $\tilde{b}$ with decreasing $C$. Fig. 3 shows joint probability distributions for $\tilde{b}$ and $\tilde{b}_{\mathrm{n}}$, with both variables centered around $\tilde{b}$ 's mean and normalized to $\tilde{b}$ 's standard deviation. The prediction $\tilde{b}_{\mathrm{n}}$ significantly overestimates $\tilde{b}$ for $C=10$, but is quite accurate for $C \leq 1$. Fig. 4 shows that the relative mean-square error $\left\langle\left(\tilde{b}_{\mathrm{n}}-\tilde{b}\right)^{2}\right\rangle /\left\langle\tilde{b}^{2}\right\rangle$ stays below $C^{2}$, quantitatively demonstrating the expected convergence. In contrast, as expected for strong turbulence, the relative mean-square error of the $i \delta$-like linear prediction
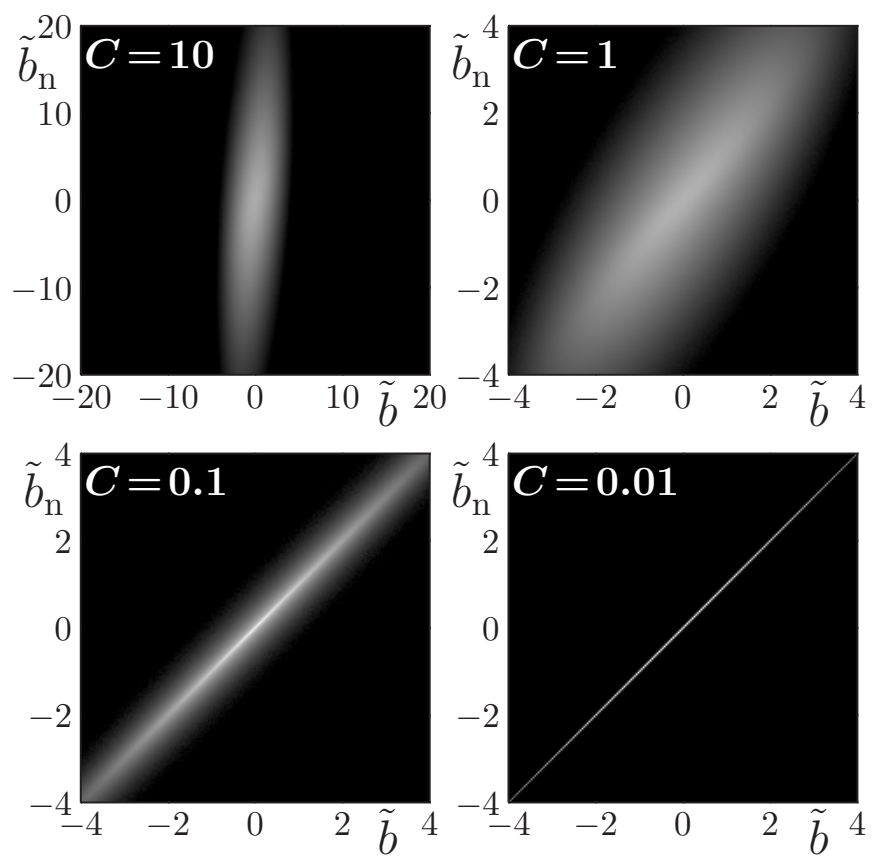

Figure 3. Joint probability distributions of $\tilde{b}$ and $\tilde{b}_{\mathrm{n}}$, using a common logarithmic grayscale with a span of $10^{6}$ between white and black. A diagonal line indicates $\tilde{b}_{\mathrm{n}}=\tilde{b}$.

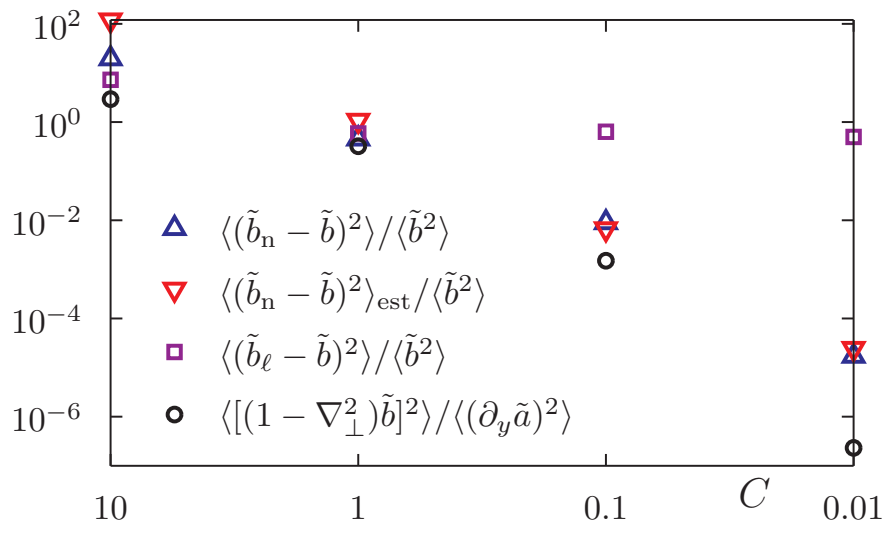

Figure 4. Normalized error of the nonadiabatic prediction $\left\langle\left(\tilde{b}_{\mathrm{n}}-\tilde{b}\right)^{2}\right\rangle /\left\langle\tilde{b}^{2}\right\rangle$ and of the comparable linear prediction $\left\langle\left(\tilde{b}_{\ell}-\right.\right.$ $\left.\tilde{b})^{2}\right\rangle /\left\langle\tilde{b}^{2}\right\rangle$, along with the normalized error estimate $\left\langle\left(\tilde{b}_{\mathrm{n}}-\right.\right.$ $\left.\tilde{b})^{2}\right\rangle_{\text {est }} /\left\langle\tilde{b}^{2}\right\rangle$ (c.f. end of Sec. V) and relative nonadiabaticity measure $\left\langle\left[\left(1-\nabla_{\perp}^{2}\right) \tilde{b}\right]^{2}\right\rangle /\left\langle\left(\partial_{y} \tilde{a}\right)^{2}\right\rangle$.

$\left\langle\left(\tilde{b}_{\ell}-\tilde{b}\right)^{2}\right\rangle /\left\langle\tilde{b}^{2}\right\rangle$ remains order-unity at small $C$. The error estimate $\left\langle\left(\tilde{b}_{\mathrm{n}}-\tilde{b}\right)^{2}\right\rangle_{\text {est }}$ from the end of Sec. V is seen to be a good predictor of $\left\langle\left(\tilde{b}_{\mathrm{n}}-\tilde{b}\right)^{2}\right\rangle$ for $C \leq 1$. Finally, the ratio $\left\langle\left[\left(1-\nabla_{\perp}^{2}\right) \tilde{b}\right]^{2}\right\rangle /\left\langle\left(\partial_{y} \tilde{a}\right)^{2}\right\rangle$ indeed well satisfies the rigorous bound given by Eq. (7).

\section{CONCLUSIONS}

Beginning with the paradigmatic Hasegawa-Wakatani equations, a unique decomposition of the electrostatic po- 
tential $\varphi$ into adiabatic $(a)$ and nonadiabatic $(b)$ portions was identified in Eqs. (3), defining $a$ and $b$ such that $a$ neither drives nor responds to the parallel current $j_{\|}$. The adiabatic variable $a$ evolves according to the HasegawaMima equation with additional terms $\propto b$ incorporating the nonadiabatic physics, including energy sources and sinks and the $\boldsymbol{E} \times \boldsymbol{B}$ nonlinearity [Eq. (4a)]. The form of the $a, b$ decomposition is independent of the type of parallel impedance, depending only on the ion polarization physics. At low $k_{\perp}$, the decomposition reduces to $a \approx n, b \approx \varphi-n$, highlighting the fact that the low- $k_{\perp}$ electron adiabatic response controls $\varphi$ without influencing $n$, as is explained in Sec. II. Recasting the energy and $\mathcal{N}_{i}$ nonlinear invariants of the HWEs in the $a$ and $b$ variables allowed a rigorous bound on the relative amplitude of nonadiabatic fluctuations for nondecaying turbulence [Eq. (7)], while also demonstrating a dissipative constraint linking the spectra of $a$ and $b$ [Eq. (6)]. The vorticity nonlinearity was found to cause energy transfer between $a$ and $b$ [Eqs. (5a) and (5b)], providing physical intuition for the self-sustained drift wave turbulence observed in Refs. 11 and 13, in which the nonlinear instability was seen to follow from an incoherent spreading of the $n, \varphi$ phase shift by the vorticity nonlinearity. The dissipation of $a$ must be independent of $b$ in order that it not act as a source term for mean-squared fluctuating ion gyrocenter density $\mathcal{N}_{i}$ (Appendix A), an important constraint on the form of purely dissipative operators for the HWEs.

In the limit of weak parallel resistivity $C \ll 1$, the transformation to the $a, b$ variables enabled the derivation of a noniterative asymptotic functional relation $\tilde{b} \approx$ $\tilde{b}_{\mathrm{n}}(a)$, both retaining [Eq. (B3)] and discarding [Eq. (9)] the contribution of zonal $\bar{b}$. Unlike $i \delta$ approximations, the prediction $\tilde{b}_{\mathrm{n}}$ retains the contribution of the vorticity nonlinearity, which is important since tokamak edge turbulence is typically strong. Since iteration is not required, the predicted functional form of $\tilde{b}_{\mathrm{n}}(a)$ is much simpler than the results of analogous calculations that do not employ the $a, b$ variables. ${ }^{31,32}$ For the $C \ll 1$ limit, this relation provides a one-field approximation to the HWEs, enhancing physical intuition and greatly easing the burden on any further analytical manipulations, such as statistical closures. Direct numerical simulation of the HWEs demonstrated that the approximation $\tilde{b}_{\mathrm{n}}(a)$ indeed rapidly converges to the dynamically evolved $\tilde{b}$ for $C \leq 1$ (Figs. 3 and 4 ), while an $i \delta$-like linear approximation has order-unity errors at all $C$.

Since the underlying physics (Sec. II) occurs quite generically in tokamak edge turbulence, the $a, b$ decomposition may also be fruitfully applied to more general problems incorporating electromagnetic fluctuations or an X-point geometry, as will be presented in upcoming publications.

\section{ACKNOWLEDGEMENTS}

Helpful discussions with Greg Hammett, Jim Myra, and Stewart Zweben are gratefully acknowledged. This work was performed in part at PPPL (supported by a Department of Energy Fusion Energy Sciences Fellowship, a National Science Foundation Graduate Research Fellowship, and the U.S. Department of Energy Contract No. DE-AC02-76-CHO-3073) and in part at the MaxPlanck-Institut für Plasmaphysik, both in Garching (supported by a Humboldt Research Fellowship for Postdoctoral Researchers) and in Greifswald (funded by the European Atomic Energy Community, with the work therefore subject to the provisions of the Euratom Treaty).

\section{Appendix A: Dissipation of Invariants}

In the $n, \varphi$ variables, the HW energy and meansquared ion gyrocenter density invariants $\mathcal{E}$ and $\mathcal{N}_{i}$ are $\frac{1}{2}\left\langle n^{2}+\left|\nabla_{\perp} \varphi\right|^{2}\right\rangle$ and $\frac{1}{2}\left\langle\left(n-\nabla_{\perp}^{2} \varphi\right)^{2}\right\rangle$. As additional non- $j_{\|}$dissipation, one is tempted to use simple diffusion on $n$ and $\nabla_{\perp}^{2} \varphi$, adding the terms $+D_{n} \nabla_{\perp}^{2} n$ and $+D_{\varphi} \nabla_{\perp}^{4} \varphi$ (with $D_{n}$ and $D_{\varphi}$ positive constants) to the RHS of Eqs. (2a) and (2b), respectively. Surprisingly, although this causes positive-definite energy dissipation, it acts in some cases as a source for $\mathcal{N}_{i}$. To see this, consider the special case $n=c_{1} \nabla_{\perp}^{2} \varphi$ with unspecified constant $c_{1}$, in which the diffusion terms jointly contribute a term $-\left(c_{1}-1\right)\left(c_{1} D_{n}-D_{\varphi}\right)\left\langle\left|\nabla_{\perp}\left(\nabla_{\perp}^{2} \varphi\right)\right|^{2}\right\rangle$ to $\partial_{t} \frac{1}{2}\left\langle\left(n-\nabla_{\perp}^{2} \varphi\right)^{2}\right\rangle$. Since the quantity under the average is positive semidefinite, this term is dissipative if and only if $\left(c_{1}-1\right)\left(c_{1} D_{n}-D_{\varphi}\right) \geq 0$. Whenever $D_{n} \neq D_{\varphi}$, values of $c_{1}$ between 1 and $D_{\varphi} / D_{n}$ make this product negative, showing the diffusion to act in those cases as a source for $\mathcal{N}_{i}$. This is undesirable for two reasons: First, $\mathcal{N}_{i}$, physically the mean-squared ion gyrocenter density fluctuation, ${ }^{37}$ should presumably be dissipated by the dominant non- $j_{\|}$dissipation mechanisms: ion-ion collisions and ion Landau damping. Second, the dissipation operators should certainly not act as sources in the common case that they are made artificially large to remove energy and $\mathcal{N}_{i}$ at grid and/or box scales in numerical simulations.

What is then the most general linear operator $\mathcal{D}$ that is guaranteed to dissipate both energy and $\mathcal{N}_{i}$ ? With $\varpi \doteq \nabla_{\perp}^{2} \varphi$ denoting the vorticity, the most general linear operator takes the form

$$
\begin{array}{r}
\partial_{t} n+\{\varphi, n\}+\omega_{n} \partial_{y} \varphi-\nabla_{\|} j_{\|}=-\left[\mathcal{D}_{n n}(n)+\mathcal{D}_{n \varpi}(\varpi)\right], \\
\partial_{t} \varpi+\{\varphi, \varpi\}-\nabla_{\|} j_{\|}=-\left[\mathcal{D}_{\varpi n}(n)+\mathcal{D}_{\varpi \varpi}(\varpi)\right],
\end{array}
$$

where $\mathcal{D}_{n n}, \mathcal{D}_{n \varpi}, \mathcal{D}_{\varpi n}$, and $\mathcal{D}_{\varpi \varpi}$ are linear operators acting on the given functions. The evolution of $\mathcal{N}_{i}$ may 
be written as

$$
\begin{array}{r}
\partial_{t} \frac{1}{2}\left\langle(n-\varpi)^{2}\right\rangle+\omega_{n}\left\langle n \partial_{y} \varphi\right\rangle=-\left\langle(n-\varpi) \mathcal{D}_{\Delta}(n-\varpi)\right\rangle \\
+\left\langle(n-\varpi)\left(\mathcal{D}_{\varpi \varpi}-\mathcal{D}_{n \varpi}-\mathcal{D}_{\Delta}\right)(\varpi)\right\rangle, \quad(\mathrm{A} 2)
\end{array}
$$

in which $\mathcal{D}_{\Delta} \doteq \mathcal{D}_{n n}-\mathcal{D}_{\varpi n}$. If $\mathcal{D}_{\varpi \varpi}-\mathcal{D}_{n \varpi}-\mathcal{D}_{\Delta} \neq 0$, then one may consider a $\varpi$ such that $\left(\mathcal{D}_{\varpi \varpi}-\mathcal{D}_{n \varpi}-\right.$ $\left.\mathcal{D}_{\Delta}\right)(\varpi) \neq 0$ and an $n=\varpi+c_{1}\left(\mathcal{D}_{\varpi \varpi}-\mathcal{D}_{n \varpi}-\mathcal{D}_{\Delta}\right)(\varpi)$ for $c_{1}$ a positive constant, in which case $\left\langle(n-\varpi)\left(\mathcal{D}_{\varpi \varpi}-\right.\right.$ $\left.\left.\mathcal{D}_{n \varpi}-\mathcal{D}_{\Delta}\right)(\varpi)\right\rangle=c_{1}^{-1}\left\langle(n-\varpi)^{2}\right\rangle$ may always be made larger than $\left\langle(n-\varpi) \mathcal{D}_{\Delta}(n-\varpi)\right\rangle$ by choosing $c_{1}$ small enough. To guarantee positive dissipation of $\mathcal{N}_{i}$, one thus requires $\mathcal{D}_{\varpi \varpi}-\mathcal{D}_{n \varpi}=\mathcal{D}_{\Delta}$. Defining the adjoint of a linear operator $\mathcal{L}$ in the usual way such that $\left\langle f_{2} \mathcal{L}^{\dagger} f_{1}\right\rangle=$ $\left\langle f_{1} \mathcal{L} f_{2}\right\rangle$, positive dissipation of $\mathcal{N}_{i}$ is then guaranteed if and only if the symmetrized form $\left(\mathcal{D}_{\Delta}+\mathcal{D}_{\Delta}^{\dagger}\right) / 2$ is a positive operator. Transforming to the $a, b$ variables, one has the additional dissipative terms for Eqs. (4):

$$
\begin{aligned}
\partial_{t}\left(1-\nabla_{\perp}^{2}\right) a+\cdots & =-\mathcal{D}_{a}(a), \\
\partial_{t} \nabla_{\perp}^{2}\left(1-\nabla_{\perp}^{2}\right) b+\cdots & =-\mathcal{D}_{a b}(a)-\mathcal{D}_{b}\left(\nabla_{\perp}^{2} b\right),
\end{aligned}
$$

in which $\mathcal{D}_{a}=\mathcal{D}_{\Delta}\left(1-\nabla_{\perp}^{2}\right)$, while $\mathcal{D}_{b}=\left(1-\nabla_{\perp}^{2}\right)\left(\mathcal{D}_{n n}+\right.$ $\left.\mathcal{D}_{\varpi \varpi}-\mathcal{D}_{\Delta}\right)$ and $\mathcal{D}_{a b}$ are unconstrained by the requirement of positive dissipation of $\mathcal{N}_{i}$. The energy evolution of Eqs. (5a) and (5b) is generalized to

$$
\begin{aligned}
\partial_{t} \frac{1}{2}\left\langle a^{2}\right. & \left.+\left|\nabla_{\perp} a\right|^{2}+\left|\nabla_{\perp} b\right|^{2}+\left(\nabla_{\perp}^{2} b\right)^{2}\right\rangle+\cdots \\
= & -\left\langle a \mathcal{D}_{a} a\right\rangle-\left\langle b \mathcal{D}_{b}\left(-\nabla_{\perp}^{2}\right) b\right\rangle+\left\langle b \mathcal{D}_{a b} a\right\rangle .
\end{aligned}
$$

Considering fluctuations with $b=0$ and with $a=0$, one sees that $\mathcal{D}_{a}+\mathcal{D}_{a}^{\dagger}$ and $\mathcal{D}_{b}\left(-\nabla_{\perp}^{2}\right)+\left(-\nabla_{\perp}^{2}\right) \mathcal{D}_{b}^{\dagger}$ must be positive operators. Additionally, one must have $\left|\left\langle b \mathcal{D}_{a b} a\right\rangle\right| \leq\left\langle a \mathcal{D}_{a} a\right\rangle+\left\langle b \mathcal{D}_{b}\left(-\nabla_{\perp}^{2}\right) b\right\rangle$ for all $a$ and $b$. This is obviously true for $\mathcal{D}_{a b}=0$, the choice made in the main text, which allows the energy dissipation operator $\left(\left(\mathcal{D}_{a}, 0\right),\left(-\mathcal{D}_{a b},-\mathcal{D}_{b} \nabla_{\perp}^{2}\right)\right)$ to be self-adjoint. It can also be straghtforwardly verified in other practical cases. For example, if $\mathcal{D}_{a}, \mathcal{D}_{b}$, and $\mathcal{D}_{a b}$ all diagonalize on Fourier modes, then let $d_{a a}\left(\boldsymbol{k}_{\perp}\right), d_{b b}\left(\boldsymbol{k}_{\perp}\right)$, and $d_{a b}\left(\boldsymbol{k}_{\perp}\right)$ be the coefficients of their Fourier transforms, for which one must have $\operatorname{Re}\left(d_{a b} \hat{b}_{\boldsymbol{k}}^{*} \hat{a}_{\boldsymbol{k}}\right) \leq \operatorname{Re}\left(d_{a a}\right)\left|\hat{a}_{\boldsymbol{k}}\right|^{2}+\operatorname{Re}\left(d_{b b}\right) k_{\perp}^{2}\left|\hat{b}_{\boldsymbol{k}}\right|^{2}$ for all $\boldsymbol{k}_{\perp}, \hat{a}_{\boldsymbol{k}}$, and $\hat{b}_{\boldsymbol{k}}$, which is true if and only if $\operatorname{Re}\left(d_{a a}\right), \operatorname{Re}\left(d_{b b}\right) \geq 0$ and $\left|d_{a b}\right|^{2} \leq 4 k_{\perp}^{2} \operatorname{Re}\left(d_{a a}\right) \operatorname{Re}\left(d_{b b}\right)$ for all $\boldsymbol{k}_{\perp} \cdot{ }^{63}$

\section{Appendix B: Near-Adiabatic Approximation with Zonal $b$}

In this appendix, Sec. $\mathrm{V}$ is straightforwardly generalized to retain $\bar{b}$, the zonal component of $b$. The fact that the parallel gradient vanishes for the zonal component implies that $\bar{b}$ is undamped by the parallel current, requiring its retention as a dynamical variable. The nonzonal portion $\tilde{b}$ may again be approximated, this time as a function of both $a$ and $\bar{b}$. The calculation is again restricted to the $2 \mathrm{D}$ formulation.
Begin with Eq. (4a) and the zonal average of Eq. (4b), noting that $\{\bar{\psi}, \bar{\xi}\}=\overline{\{\bar{\psi}, \widetilde{\xi}\}}=0$ for arbitrary functions $\psi$ and $\xi$ :

$$
\begin{aligned}
& \partial_{t}\left(1-\nabla_{\perp}^{2}\right) a+\omega_{n} \partial_{y} \tilde{a}-\left\{a, \nabla_{\perp}^{2} a\right\}+\left(\partial_{x} \bar{b}\right) \partial_{y}\left(1-\nabla_{\perp}^{2}\right) \tilde{a} \\
&+\mathcal{D}_{a} a=-\omega_{n} \partial_{y} \tilde{b}-\left\{\tilde{b},\left(1-\nabla_{\perp}^{2}\right) a\right\}, \text { (B1a) } \\
& \partial_{t} \partial_{x}^{2}\left(1-\partial_{x}^{2}\right) \bar{b}+\overline{\left\{\tilde{a}, \nabla_{\perp}^{2} \tilde{a}\right\}}=-\overline{\left\{\tilde{b}, \nabla_{\perp}^{2} \tilde{a}\right\}} \\
&-\left(1-\partial_{x}^{2}\right) \overline{\left\{\tilde{a}+\tilde{b}, \nabla_{\perp}^{2} \tilde{b}\right\}}-\partial_{x}^{2} \overline{\{\tilde{a}, \tilde{b}\} .} \quad(\text { B1b) }
\end{aligned}
$$

The dynamical equation for $\tilde{b}$ is simply the difference of Eqs. (4b) and (B1b). Just as in Sec. V, Eq. (4c) implies that $\tilde{b} / j_{\|} \sim O(C)$. The zeroth-order equation for $\tilde{b}$ thus reduces to

$$
\begin{aligned}
& \left(1-\nabla_{\perp}^{2}\right) \nabla_{\|} j_{\|} \approx-\omega_{n} \partial_{y} \nabla_{\perp}^{2} \tilde{a}+\left\{\widehat{a, \nabla_{\perp}^{2} a}\right\} \\
& \quad-\left(2-\nabla_{\perp}^{2}\right)\left[\left(\partial_{y} \tilde{a}\right)\left(\partial_{x}^{3} \bar{b}\right)\right]-2\left(\partial_{x} \partial_{y} \tilde{a}\right)\left(\partial_{x}^{2} \bar{b}\right),
\end{aligned}
$$

quite similar to Eq. (8). One may again obtain the leading-order $\tilde{b}$ by substituting this result into Eq. (4c), obtaining

$$
\begin{aligned}
\tilde{b} & \approx C\left(1-\nabla_{\perp}^{2}\right)^{-1} \nabla_{\|}^{-2}\left(1-\nabla_{\perp}^{2}\right)^{-1}\left[\omega_{n} \partial_{y} \nabla_{\perp}^{2} \tilde{a}-\left\{\widehat{\left.a, \nabla_{\perp}^{2} a\right\}}\right.\right. \\
& \left.+\left(2-\nabla_{\perp}^{2}\right)\left[\left(\partial_{y} \tilde{a}\right)\left(\partial_{x}^{3} \bar{b}\right)\right]+2\left(\partial_{x} \partial_{y} \tilde{a}\right)\left(\partial_{x}^{2} \bar{b}\right)\right] \cdot \quad \text { (B3) }
\end{aligned}
$$

Since the leading-order $\tilde{b}$ is already $O(C)$ relative to $a$ and $\bar{b}$, and since $j_{\|}$does not appear explicitly in either Eq. (B1a) or (B1b), one may obtain a first-orderaccurate model simply by substituting the formula given by Eq. (B3) for the appearances of $\tilde{b}$ in Eqs. (B1).

\section{Appendix C: Numerical Convergence}

In order to achieve converged numerical results despite the very slow saturation of the turbulent spectra for $C \ll$ 1 , a resolution-doubling procedure was used to improve computational efficiency. Details of this scheme and of convergence tests are the theme of this appendix.

The temporal evolution of the spectra depends strongly on $C$. For $C \gtrsim 1$, the density and potential spectra saturate rapidly $\left(\sim O\left(10^{2}\right)\right.$ normalized times $)$, then fluctuate around fixed values. For $C \ll 1$, the density fluctuation spectrum initially develops a steeplysloped region (decreasing with increasing $k_{\perp}$ ), first at high $k_{\perp}$ then building up towards lower $k_{\perp}$. Once the spectral peak at the low- $k_{\perp}$ edge of this region reaches below around $k_{\perp} \sim 0.1$, its motion slows greatly and the $k_{\perp} \gtrsim 0.1$ spectrum broadens, concurrent with a much more active energy source and sink near $k_{\perp} \approx 1$. After this point, the spectral shape stays roughly fixed as the overall amplitude grows very slowly to its saturated value. This behavior persisted essentially unchanged for increasing domain sizes up to $\left(640 \pi \rho_{s}\right)^{2}$ (at $C=0.1$ with a fixed grid spacing of around $0.98 \rho_{s}$ ), thus appears not 
to be a box-size effect. [Similar behavior was observed in simulations of HM turbulence omitting the linear term, ${ }^{64}$ thus it presumably follows from the nonlinear structure of the equations when $n \approx \varphi$.] The fluctuation energy $\mathcal{E}$ eventually saturates at a level that is strongly dependent on $\nu$ (increasing with increasing $\nu$ ), despite the fact that $\mathcal{E}$ is often dominated by low- $k_{\perp}$ contributions from $\left\langle n^{2}\right\rangle$.

In order to check domain size convergence at each $C \geq 0.1$, reference runs with $\left(80 \pi \rho_{s}\right)^{2}$ box size and $0.98 \rho_{s}$ grid spacing were run to saturation. Four copies of a snapshot of the saturated state were set side-by-side as the initial state of a $\left(160 \pi \rho_{s}\right)^{2}$ run. The resulting saturation levels and spectra were virtually unchanged by the doubling. However, box sizes of $\left(40 \pi \rho_{s}\right)^{2}$ were sometimes inadequate, losing the low- $k_{\perp}$ rolloff of the $\left\langle n^{2}\right\rangle$ spectrum.

The hyperviscosity $\nu$ was chosen such that the resulting damping was larger than the energy source and resistive sink for roughly the last factor of two in large $k_{\perp}$, which resulted in a relatively steep dropoff of the fluctuation spectra in the same $k_{\perp}$ range. As a further test, snapshots of saturated reference runs for $C \geq 0.1$ were used as the initial conditions for $0.49 \rho_{s}$ grid spacing runs at the same domain size, linearly interpolating onto the new grid points. Only modest changes were observed in saturation levels or spectra, the latter of which decayed steeply for fluctuations smaller than the original grid scale.

To obtain resolution convergence, snapshots of saturated reference runs were again linearly interpolated onto grids with halved $\left(0.49 \rho_{s}\right)$ spacing, but now used as initial conditions for runs with $\nu$ reduced by a factor of 16 . After the subsequent run saturated, the resolution refinement procedure was repeated until saturation amplitudes and spectra were no longer significantly affected. The data for Figs. 3 and 4 were drawn from the concluding saturated, high-resolution periods. A subsequent additional resolution refinement and short run at each $C \geq 0.1$ showed no significant changes from the plotted values (cf. Fig. 6). Control tests performed for $C=10$ and 1 , starting with the standard initial conditions in the high-resolution box and running to saturation, also reproduced the results of the resolution-refinement runs.

The resolution refinements typically reduced $\left\langle n^{2}\right\rangle$ while increasing vorticity $\left\langle\left(\nabla_{\perp}^{2} \varphi\right)^{2}\right\rangle$ and without significantly affecting $\left\langle\left|\nabla_{\perp} \varphi\right|^{2}\right\rangle$, by reducing fluctuation amplitudes only at low $k_{\perp}$ while increasing higher- $k_{\perp}$ activity. The observed drop in $\left\langle n^{2}\right\rangle$ was particularly strong for $C=0.1$, for which the reference runs saturated at $\left\langle n^{2}\right\rangle \sim O\left(10^{2}\right)$, with $\left\langle n^{2}\right\rangle$ dropping by about an order of magnitude due to the resolution refinements. Presumably, the increased high- $k_{\perp}$ and nonadiabatic fluctuations allowed by the higher resolution and lower $\nu$ allowed a stronger direct cascade contribution to draw energy out of the lower- $k_{\perp}$ $\left\langle n^{2}\right\rangle$. It is important to note that, even at low $C$, the $\boldsymbol{E} \times \boldsymbol{B}$ energy $\left\langle\left|\nabla_{\perp} \varphi\right|^{2}\right\rangle$ remained $\sim O(1)$ for both reference and refined runs. Since the nonlinearities depend only on perpendicular gradients of $\varphi$ and $(\varphi-n)$, they

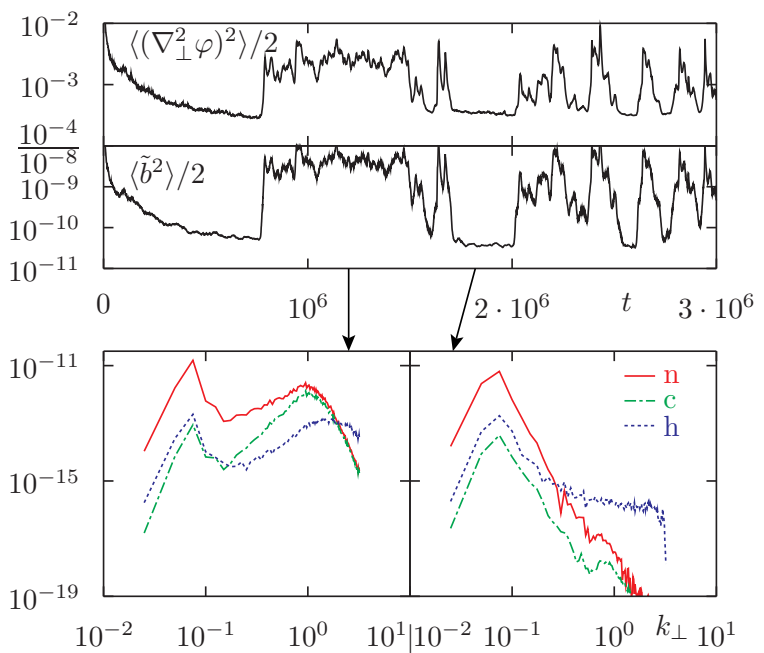

Figure 5. Vorticity and nonadiabatic fluctuation levels switching between higher and lower amplitude states, concurrent with the appearance and disappearance of the $k_{\perp} \approx 1$ feature in the spectra of the energy source (n) and resistive (c) and hyperviscous (h) sink spectra.

did not exceed order-unity for $C \ll 1$ despite the large $\left\langle n^{2}\right\rangle$.

Because of the very long run times required, the $C=$ 0.01 reference run was started with an initial amplitude about 6.7 times larger than the other cases, and was refined before $\left\langle n^{2}\right\rangle$ saturated, although after all other time traces seemed fairly constant. [Control tests showed that, after a transient period, the fluctuation amplitudes and spectra are in fact independent of the initial magnitude of $\left\langle n^{2}\right\rangle$.] All quantities plotted in Fig. 4 had reached approximately steady-state values before the end of the reference runs, as evidenced by their time traces, which became fairly constant when the other non- $\left\langle n^{2}\right\rangle$ time traces did. The plotted quantities were similarly unaffected by the evolution of $\left\langle n^{2}\right\rangle$ in the refined runs.

In fact, the amplitude of nonadiabatic fluctuation $\left\langle\tilde{b}^{2}\right\rangle$ and of the error $\left\langle\left(\tilde{b}-\tilde{b}_{\mathrm{n}}\right)^{2}\right\rangle$ was most strongly affected by the presence or absence of the $k_{\perp} \approx 1$ feature in the energy source and sink terms, which also seemed to control the vorticity level, as shown in Fig. 5. The $k_{\perp} \approx 1$ energy feature only developed later in the $C<1$ runs, and not at all when $\nu$ was too large. In intermediate- $\nu$ cases, the $k_{\perp} \approx 1$ feature sometimes switched on and off irregularly. In order to concurrently allow the $k_{\perp} \approx 1$ feature and maintain adequate dissipation at the grid scale, finer grid spacing may sometimes be required.

Although $\left\langle\tilde{b}^{2}\right\rangle$ and $\left\langle\left(\tilde{b}-\tilde{b}_{\mathrm{n}}\right)^{2}\right\rangle$ changed somewhat for grid refinements after $0.49 \rho_{s}$, their ratio $\left\langle\left(\tilde{b}-\tilde{b}_{\mathrm{n}}\right)^{2}\right\rangle /\left\langle\tilde{b}^{2}\right\rangle$ changed very little (Fig. 6 ), thus Figs. 3 and 4 are quite well converged for the final resolutions of $0.12 \rho_{s}, 0.12 \rho_{s}$, and $0.25 \rho_{s}$ at $C=10,1$, and 0.1 , respectively, while $C=0.01$ appears adequately resolved at $0.49 \rho_{s}$. 


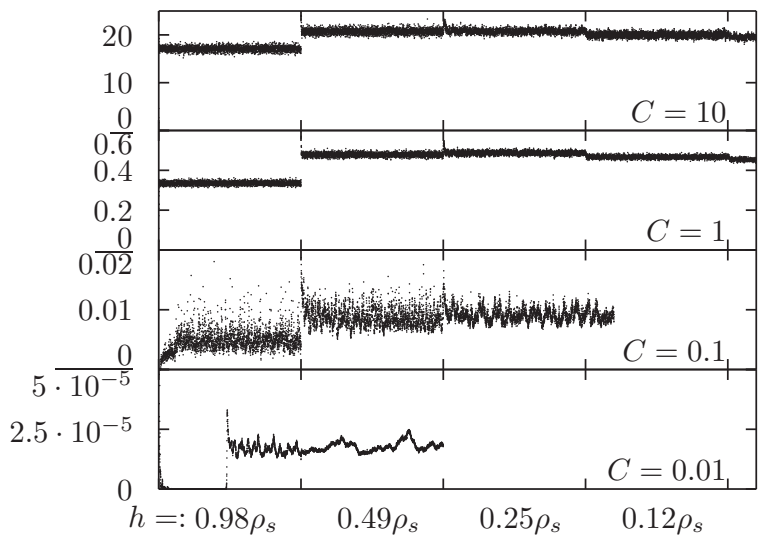

Figure 6. Time traces of $\left\langle\left(\tilde{b}_{\mathrm{n}}-\tilde{b}\right)^{2}\right\rangle /\left\langle\tilde{b}^{2}\right\rangle$ for $C=10,1,0.1$ and 0.01 . The time axis is distended such that resolution refinements are evenly spaced, and labeled with the dimensional grid spacing $h$. Traces from short additionally-refined runs with grid spacing of $0.06 \rho_{s}, 0.06 \rho_{s}$, and $0.12 \rho_{s}$ for $C=10,1$, and 0.1 are included.

\section{REFERENCES}

${ }^{1}$ F. Ryter, F. Leuterer, G. Pereverzev, H.-U. Fahrbach, J. Stober, W. Suttrop, and ASDEX Upgrade Team, Phys. Rev. Lett. 86, 2325 (2001).

${ }^{2}$ G. Tardini, A. Peeters, G. Pereverzev, F. Ryter, J. Stober, and ASDEX Upgrade Team, Nucl. Fusion 42, 258 (2002).

${ }^{3}$ M. Kotschenreuther, W. Dorland, M. A. Beer, and G. W. Hammett, Phys. Plasmas 2, 2381 (1995).

${ }^{4}$ A. M. Dimits, B. Cohen, N. Mattor, W. Nevins, D. Shumaker, S. Parker, and C. Kim, Nucl. Fusion 40, 661 (2000).

${ }^{5}$ F. Jenko, W. Dorland, and G. W. Hammett, Phys. Plasmas 8, 4096 (2001).

${ }^{6}$ W. Suttrop, M. Kaufmann, H. J. de Blank, B. Brüsehaber, K. Lackner, V. Mertens, H. Murmann, J. Neuhauser, F. Ryter, H. Salzmann, J. Schweinzer, J. Stober, H. Zohm, and the ASDEX Upgrade Team, Plasma Phys. Controlled Fusion 39, 2051 (1997), Figs. 8 and 9.

${ }^{7}$ F. Wagner, G. Becker, K. Behringer, D. Campbell, A. Eberhagen, W. Engelhardt, G. Fussmann, O. Gehre, J. Gernhardt, G. v. Gierke, G. Haas, M. Huang, F. Karger, M. Keilhacker, O. Klüber, M. Kornherr, K. Lackner, G. Lisitano, G. G. Lister, H. M. Mayer, D. Meisel, E. R. Müller, H. Murmann, H. Niedermeyer, W. Poschenrieder, H. Rapp, H. Röhr, F. Schneider, G. Siller, E. Speth, A. Stäbler, K. H. Steuer, G. Venus, O. Vollmer, and Z. Yü, Phys. Rev. Lett. 49, 1408 (1982).

${ }^{8}$ J. W. Connor and H. R. Wilson, Plasma Phys. Controlled Fusion 42, R1 (2000).

${ }^{9}$ F. Wagner, Plasma Phys. Controlled Fusion 49, B1 (2007).

${ }^{10}$ B. D. Scott, Phys. Plasmas 12, 062314 (2005).

${ }^{11}$ B. D. Scott, Phys. Rev. Lett. 65, 3289 (1990).

${ }^{12}$ A. Zeiler, D. Biskamp, J. F. Drake, and P. N. Guzdar, Phys. Plasmas 3, 2951 (1996)

${ }^{13}$ B. D. Scott, New J. Phys. 4, 52 (2002).

${ }^{14}$ S. J. Zweben, J. A. Boedo, O. Grulke, C. Hidalgo, B. LaBombard, R. J. Maqueda, P. Scarin, and J. L. Terry, Plasma Phys. Controlled Fusion 49, S1 (2007), Sec. 5.3, and references therein. ${ }^{15}$ B. D. Scott, Plasma Phys. Controlled Fusion 45, A385 (2003). ${ }^{16}$ B. Scott, Phys. Plasmas 5, 2334 (1998).

${ }^{17}$ J. M. Albert, P. L. Similon, and R. N. Sudan, Phys. Fluids B 2, 3032 (1990)

${ }^{18}$ A. Hasegawa and M. Wakatani, Phys. Rev. Lett. 50, 682 (1983). ${ }^{19}$ M. Wakatani and A. Hasegawa, Phys. Fluids 27, 611 (1984).

${ }^{20}$ P. W. Terry and P. H. Diamond, Phys. Fluids 28, 1419 (1985).
${ }^{21}$ F. Y. Gang, P. H. Diamond, J. A. Crotinger, and A. E. Koniges, Phys. Fluids B 3, 955 (1991).

${ }^{22}$ D. A. Baver, P. W. Terry, R. Gatto, and E. Fernandez, Phys. Plasmas 9, 3318 (2002).

${ }^{23}$ G. Hu, J. A. Krommes, and J. C. Bowman, Phys. Plasmas 4, 2116 (1997).

${ }^{24}$ A. Hasegawa and K. Mima, Phys. Rev. Lett. 39, 205 (1977).

${ }^{25}$ W. Horton, Phys. Rev. Lett. 37, 1269 (1976).

${ }^{26} \mathrm{P}$. Terry and W. Horton, Phys. Fluids 25, 491 (1982).

${ }^{27}$ R. E. Waltz, Phys. Fluids 26, 169 (1983).

${ }^{28}$ G. G. Craddock, A. E. Koniges, J. A. Crotinger, P. H. Diamond, D. E. Newman, and P. W. Terry, Phys. Plasmas 1, 1877 (1994).

${ }^{29}$ M. Kono and E. Miyashita, Phys. Fluids 31, 326 (1988).

${ }^{30}$ Y.-M. Liang, P. H. Diamond, X.-H. Wang, D. E. Newman, and P. W. Terry, Phys. Fluids B 5, 1128 (1993).

${ }^{31}$ J. A. Crotinger and T. H. Dupree, Phys. Fluids B 4, 2854 (1992).

${ }^{32}$ V. Naulin and K. H. Spatschek, Phys. Rev. E 55, 5883 (1997).

${ }^{33}$ The normalized density and temperature that appear in the HWEs are very similar: $n \doteq\left[\left(n_{e}-n_{e 0}\right) / n_{e 0}\right]\left(L_{\perp} / \rho_{s}\right)$ and $\varphi \doteq\left(e \phi / T_{e 0}\right)\left(L_{\perp} / \rho_{s}\right)$.

${ }^{34}$ B. Scott, Plasma Phys. Controlled Fusion 39, 1635 (1997).

${ }^{35}$ B. Scott, Phys. Lett. A 320, 53 (2003).

${ }^{36}$ V. Naulin, A. Kendl, O. E. Garcia, A. H. Nielsen, and J. J. Rasmussen, Phys. Plasmas 12, 052515 (2005).

${ }^{37}$ J. A. Krommes, Physics Reports 360, 1 (2002).

${ }^{38}$ R. Numata, R. Ball, and R. L. Dewar, Phys. Plasmas 14, 102312 (2007).

${ }^{39}$ T. Stoltzfus-Dueck, Tokamak Edge Turbulence and the Approach to Adiabatic Response, Ph.D. thesis, Princeton University (Apr. 2009).

${ }^{40}$ W. D. D'haeseleer, W. N. G. Hitchon, J. D. Callen, and J. L. Shohet, Flux Coordinates and Magnetic Field Structure: A Guide to a Fundamental Tool of Plasma Theory (SpringerVerlag, 1991).

${ }^{41}$ For example, the Hasegawa-Mima equation ${ }^{24}$ may be seen as an equation evolving $n-\nabla_{\perp}^{2} \varphi$ for adiabatic electrons $n=$ $\varphi$. The combination also appears in an intermediate step of the original derivation of the HWEs ${ }^{18}$ as "potential vorticity" (the most common name, harking back to analogous variables used in atmospheric dynamics ${ }^{65}$ ) in a derivation of enstrophy conservation, ${ }^{66}$ and as a passively advected quantity in statistical calculations. ${ }^{67,68}$ Equations for $n-\nabla_{\perp}^{2} \varphi$ were used as intermediate steps in the derivation of iterative near-adiabatic models, ${ }^{31,32}$ but the approximate closure was then calculated as a function of $\varphi$ rather than of $n-\nabla_{\perp}^{2} \varphi$.

${ }^{42}$ G. W. Hammett, W. Dorland, and F. W. Perkins, Phys. Fluids B 4, 2052 (1992).

${ }^{43}$ B. D. Scott, Phys. Plasmas 14, 102318 (2007).

${ }^{44}$ D. H. E. Dubin, J. A. Krommes, C. Oberman, and W. W. Lee, Phys. Fluids 26, 3524 (1983).

${ }^{45}$ P. W. Terry, D. A. Baver, and D. R. Hatch, Phys. Plasmas 16, 122305 (2009).

${ }^{46}$ The dissipation modeled by this operator is physically dominated by ion Landau damping at large perpendicular scales and ion-ion collisions (viscosity) at small perpendicular scales. ${ }^{39}$.

${ }^{47}$ R. H. Kraichnan, Phys. Fluids 10, 1417 (1967).

${ }^{48}$ The linear evolution of these nonlinear invariants must therefore be slow for nondecaying turbulence and $C \ll 1$ : As will be shortly discussed, nondecaying $\mathcal{E}$ forces $\tilde{b} / \tilde{a} \sim O(C)$, thus comparing the RHS of Eqs. (5a) and (5b) with $\mathcal{E}_{a}$, one sees that the timescale of $\mathcal{E}$ evolution (neglecting $\mathcal{D}_{a}$ ) is long, $O\left(C^{-1}\right)$. Comparison of the RHS and LHS of Eq. (5c) shows that $\mathcal{N}_{i}$ also evolves (again neglecting $\mathcal{D}_{a}$ ) on the long $O\left(C^{-1}\right)$ timescale. All other nonlinear invariants undergo linear evolution on the $O(1)$ timescale, thus are less robust when $C$ is small.

${ }^{49}$ Although the other invariants may of course also be expressed in the $a, b$ variables, we will have no need of them in the following analysis.

${ }^{50}$ B. D. Scott, H. Biglari, P. W. Terry, and P. H. Diamond, Phys. Fluids B 3, 51 (1991). 
${ }^{51}$ S. J. Camargo, D. Biskamp, and B. D. Scott, Phys. Plasmas 2, 48 (1995).

${ }^{52}$ Note that only spatially differentiated $a$ appears in Eq. (4b), thus one must in fact only require that the amplitudes of the perpendicular derivatives of $a$-not of $a$ itself - remain order unity.

${ }^{53}$ In $3 \mathrm{D}, \tilde{b} \approx \tilde{b}_{\mathrm{n}}$ does not necessarily hold for resonant or near resonant modes with $k_{\|}$small enough that $C / k_{\|}^{2} \gtrsim O(1)$.

${ }^{54}$ M. Ottaviani and J. A. Krommes, Phys. Rev. Lett. 69, 2923 (1992).

${ }^{55}$ Our 2D collisional parallel damping coefficient $k_{\|}^{2} / C$ is replaced by a Landau damping coefficient $\delta_{0}^{-1}$ in $\mathrm{CD}$ and a collisional or Landau damping coefficient $\delta^{-1}$ in NS.

${ }^{56}$ For the present analysis, $a_{k_{\perp}}$ should roughly be thought of as the rms amplitude of a band-pass-filtered $a$, retaining only fluctuations with wave numbers between $k_{\perp} / \sqrt{2}$ and $\sqrt{2} k_{\perp}$. For the second-order $\left(1-\nabla^{2}\right)\left\{b, \nabla^{2} b\right\}$ nonlinearity, one may estimate $j_{\| k_{\perp}}$ using Eq. (8). Alternatively, one may use Eq. (7) to argue that this this term is smaller than $\left(1-\nabla_{\perp}^{2}\right)\left\{a, \nabla_{\perp}^{2} b\right\}$, then bound $\left(1-\nabla_{\perp}^{2}\right)\left\{a, \nabla_{\perp}^{2} b\right\}$ relative to the $j_{\|}$term with no assumption on $j_{\| k_{\perp}}$ required.

${ }^{57}$ This approximation is considerably simplified if one uses Eq. (9) to substitute for $\left\{a \widetilde{\nabla_{\perp}^{2}} a\right\}$, thus avoiding the appearance of cubic nonlinearities.
${ }^{58}$ B. D. Scott, New J. Phys. 7, 92 (2005).

${ }^{59}$ V. Naulin and A. H. Nielsen, SIAM Journal on Scientific Computing 25, 104 (2003)

60 A. Arakawa, J. Comput. Phys. 135, 103 (1997).

${ }^{61}$ G. E. Karniadakis, M. Israeli, and S. A. Orszag, J. Comput. Phys. 97, 414 (1991).

${ }^{62}$ The $C=0.01$ case was still evolving somewhat as plot data was taken, as discussed in Appendix C.

${ }^{63}$ However, note that in order to guarantee positive-definite dissipation of the $\boldsymbol{E} \times \boldsymbol{B}$ energy $\left\langle\left|\nabla_{\perp} \varphi\right|^{2}\right\rangle / 2$ (resp. of $\left.\left\langle n^{2}\right\rangle / 2\right)$, one must set $\mathcal{D}_{\varpi n}=0$ (resp. $\left.\mathcal{D}_{n \varpi}=0\right)$, as one may show using arguments like those following Eq. (A2). The only choice guaranteeing positive-definite dissipation of all three of $\left\langle\left|\nabla_{\perp} \varphi\right|^{2}\right\rangle / 2,\left\langle n^{2}\right\rangle / 2$, and $\mathcal{N}_{i}$ is a diagonal dissipation matrix with $\mathcal{D}_{n n}=\mathcal{D}_{\varpi \varpi}$. Assuming $\mathcal{D}_{n n}$ and $\nabla_{\perp}^{2}$ commute, this is equivalent to setting $\mathcal{D}_{b}=\mathcal{D}_{a}$ and $\mathcal{D}_{a b}=0$ in Eqs. (A3).

${ }^{64}$ C. V. Tran and D. G. Dritschel, J. Fluid Mech. 551, 435 (2006). ${ }^{65}$ B. J. Hoskins, M. E. McIntyre, and A. W. Robertson, Quarterly Journal of the Royal Meteorological Society 111, 877 (1985).

${ }^{66}$ A. Hasegawa and M. Wakatani, Phys. Rev. Lett. 59, 1581 (1987).

${ }^{67}$ Y. Z. Zhang and S. M. Mahajan, Phys. Fluids B 5, 2000 (1993).

${ }^{68}$ Ö. D. Gürcan, X. Garbet, P. Hennequin, P. H. Diamond, A. Casati, and G. L. Falchetto, Phys. Rev. Lett. 102, 255002 (2009). 